\title{
DpgC-catalyzed peroxidation of DPA-CoA: insights onto the spin-forbidden transition and charge transfer mechanisms
}

\author{
Pablo Ortega, ${ }^{1}$ Alexandre Zanchet, ${ }^{1}$ Cristina Sanz-Sanz, ${ }^{2}$ Susana Gómez-Carrasco, ${ }^{1}$ Lola González-Sánchez, ${ }^{1}$ and \\ Pablo G. Jambrina ${ }^{1}$ \\ ${ }^{1)}$ Departamento de Química Física. University of Salamanca, Salamanca 37008, \\ Spain. \\ ${ }^{2)}$ Departamento de Química Física Aplicada. University Autonoma de Madrid, Madrid 28049, \\ Spain.
}

(Dated: 29 April 2020)

Oxygenases are a family of enzymes that catalyse the breaking of molecular oxygen with incorporation of, at least, one oxygen atom into an organic substrate. Since molecular oxygen is a diradical and most organic molecules have no unpaired-electrons, reactions catalysed by oxygenases involve changes in the spin state of the system that are forbidden in non-relativistic quantum theory. To overcome this limitation, oxygenases usually require metal or redox cofactors for catalysis. Intriguingly, some oxygenases can catalyse oxygen incorporation reactions even in the absence of any cofactor, but the detailed mechanism followed by these enzymes to overcome this limitation is still unknown. In the present work we give insight onto the mechanism for the enzymatic cofactor-independent oxidation of 3,5-dihydroxyphenylacetyl-CoA (DPA-CoA) by the combination of multi-reference calculations on a model system, with QM/MM calculations for the enzymatic reaction. Our results reveal that intersystem crossing takes place without requiring concerted protonation of molecular oxygen. We characterized and identified the nine concurrent electronic states, showing that a first electron transfer is concomitant with the triplet-singlet transition (intersystem crossing). The enzyme apparently plays a passive role in promoting the intersystem crossing, although spontaneous reorganization of the water-wire connecting the active site with the bulk presets the substrate for subsequent chemical transformations. We believe that our results are fairly general showing that stabilization of the singlet radical-pair state between molecular oxygen and enolates is enough to promote spin-forbidden reaction without the need of neither metal cofactors nor basic residues in the active site.

\section{INTRODUCTION}

The great oxygenation event, approximately 2.4 billion years ago, was one of the most important episodes in the history of life on Earth. ${ }^{1}$ The accumulation of molecular oxygen $\left(\mathrm{O}_{2}\right)$ in the atmosphere eventually caused the extinction of a myriad of life forms. ${ }^{2}$ Those that survived evolved to exploit the presence of $\mathrm{O}_{2}$, using it to release the energy that cells will use for its maintenance and growth. The oxygen reduction reaction is also crucial for emerging energy technologies, such as solar fuels production. ${ }^{3-5}$

From a chemical point of view, reactions between $\mathrm{O}_{2}$ and organic matter are conceptually complicated. The majority of the stable molecules involved in a biological process are singlets (all the electrons are paired). $\mathrm{O}_{2}$ is an exception to this rule, and in its ground electronic state has two unpaired electrons, it is a triplet. Hence, reactions between $\mathrm{O}_{2}$ and organic molecules should involve the change of the spin state (intersystem crossing), a process that is forbidden in non-relativistic quantum chemistry, and accordingly should be very slow at room temperature. It explains why organic matter exists in our oxidizing atmosphere.

To circumvent this limitation, enzymes typically use transition metals, mostly iron, to catalyze reactions with $\mathrm{O}_{2} \cdot{ }^{6-15}$ Transition metals also contain unpaired electrons so may react with $\mathrm{O}_{2}$ via a spin-allowed reaction. Moreover, metals can be used to preactivate the substrate, forming a radical that can react with $\mathrm{O}_{2} \cdot{ }^{6,14,16}$ Last but not least, spin-orbit coupling (SOC), ${ }^{17,18}$ the relativist effect that permits the intersystem crossing, is expected to be significantly strong for systems that include transition metals. Other proteins use redox organic cofactors to catalyze spin-forbidden reactions, especially flavin. ${ }^{19-21}$

Intriguingly, some oxygenases are capable of catalyzing the incorporation of at least one oxygen atom from $\mathrm{O}_{2}$ to an organic substrate without needing any cofactor. ${ }^{22-24}$ The mechanism followed by that enzymes to catalyze has been studied both computational and experimentally (see for example Ref. 25-38) although the procedure used to facilitate the intersystem crossing is still controversial. ${ }^{29,35}$ In this article we will try to shed light onto this process.

From among these proteins, we will focus on DpgC. DpgC was the first protein for which a crystal structure showing a substrate analogue and $\mathrm{O}_{2}$ bound to the enzyme was resolved. ${ }^{36-38} \mathrm{DpgC}$ is a hexameric crotonase oxygenase $^{39,40}$ that plays a key role in the biosynthesis of dihydroxyphenylglycine (DPG), a nonnatural amino acid found in "antibiotics of last resort" such as vancomycin or teicoplanin. ${ }^{41}$ In particular, DpgC catalyzes the cofactor-independent oxidation of 3,5-dihydroxyphenylacetyl-CoA (DPA-CoA) to 3-5dihydroxyphenyl-glyoxylate (DPGX) (Fig. 1). When DPA-CoA binds to the active site, it is deprotonated at $\mathrm{C}_{\alpha}$. Deuterium atom exchange was observed even at anaerobic conditions, confirming that this first step 


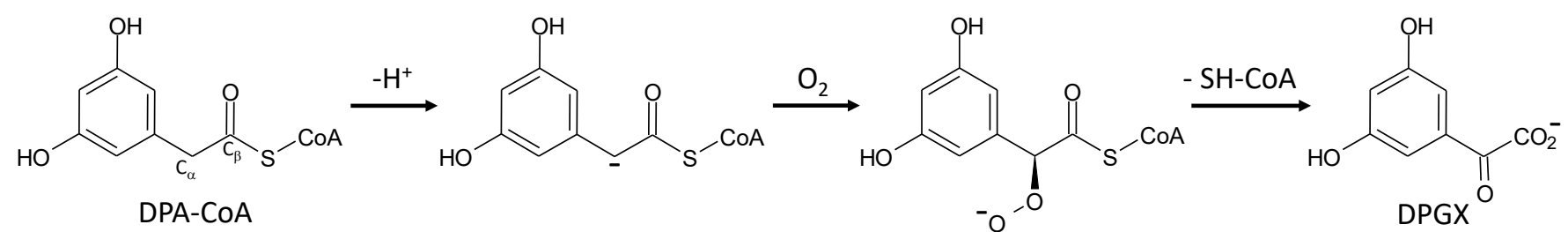

FIG. 1. Proposed mechanisms for the conversion of 3,5-dihydroxyphenylacetyl-CoA (DPA-CoA) to 3-5-dihydroxyphenylglyoxylate (DPGX) by DpgC.

is $\mathrm{O}_{2}$ independent, and that $\mathrm{DpgC}$ stabilizes the enolate before $\mathrm{O}_{2}$ access the active site. ${ }^{42}$ Following deprotonation of $\mathrm{C}_{\alpha}, \mathrm{O}_{2}$ diffuses into the active site. Molecular dynamics (MD) calculations predict that there are three main pathways for $\mathrm{O}_{2}$ diffusion, ${ }^{43}$ all of them guiding $\mathrm{O}_{2}$ to the hydrophobic pocket in which $\mathrm{O}_{2}$ was resolved in the crystal structure. These simulations also suggested that the binding of $\mathrm{O}_{2}$ to the active site is rather weak, which is compensated by a higher frequency of $\mathrm{O}_{2}$ entries into the active site. ${ }^{43}$

In the active site, $\mathrm{O}_{2}$ is located in a hydrophobic pocket. Reaction between $\mathrm{O}_{2}$ and DPA-CoA is expected to proceed via a peroxide intermediate. ${ }^{36,38}$ This is a spin-forbidden process, and it is likely the rate-limiting stage of the overall process. Contrary to what was observed for other proteins such as Glucose Oxidase ${ }^{33}$ where a His516 acts as a proton donor and protonate $\mathrm{O}_{2}$ throughout the reaction, in the $\mathrm{O}_{2}$ pocket of DpgC there are no aminoacids that could play that role. In a subsequent step, the peroxide breaks leading to the formation of DPGX via either a Criegee rearrangement or following a dioxetane intermediate. ${ }^{42}$

There are two reasons why we believe that $\mathrm{DpgC}$ is an excellent system for the characterization of the general mechanism of a spin-forbidden cofactorless addition of $\mathrm{O}_{2}$ to an organic enolate. First, the lack of basic residues around the active site permits to analyze to which extent the formation of the peroxide is possible in the absence of aminoacids that could protonate the peroxide. In DpgC we do not find aromatic residues that could be involved in $\pi-\pi$ stacking interactions, so the hypothesis of a "spin-well" could be ruled out. ${ }^{25}$ Second, the peroxide is formed outside the aromatic ring, which facilitates multireference calculations.

From a fundamental point of view, the study of spinforbidden reactions is very challenging. It requires the characterization of all the concurrent potential energy surfaces (PESs), at least two of different spin multiplicities. For these reactions, the minimum energy crossing point (MECP) between the two PESs of different spin multiplicities plays the role of the effective barrier. ${ }^{44-49}$ To determine the rate coefficient of a spin-forbidden reaction, it is also necessary to calculate the SOC. In the non-adiabatic transition state theory, the transmission coefficient is approximated by the hopping probability, which depends on the magnitude of the SOC, and also on the difference in slope of the PESs along the reac- tion coordinate in the crossing point. In its simplest case, the hopping probability is calculated using the celebrated Landau-Zener formula. ${ }^{50}$ For typical values of SOC, the hopping probability lies between 0.001 and 0.1 , which is equivalent to an increase of the activation energy of $1-4 \mathrm{kcal} / \mathrm{mol}$ at room temperature. ${ }^{47,49}$ Similar methodologies have been successfully applied to the study of some enzymatic reactions, ${ }^{15,28-33,44,51-53}$ including glucose oxidase ${ }^{32,33}$ and p-hydroxyphenylacetate hydroxylase, ${ }^{28}$ for which $\mathrm{O}_{2}$ reacts with flavin, and the cofactorless oxygenases HOD ${ }^{29,31}$ and Nogalamycin Monoxygenase, ${ }^{30}$ some of them using small active site models.

Regarding the available ab-initio methods that could be used to describe spin-forbidden reactions, if possible it is advised to use multireference methods, which permit to include several electronic states on an equal footing, and to estimate the magnitude of the SOC. From within these methods, multiconfiguration reference internally contracted configuration interaction (MRCI) method is one of the most accurate, and it is considered the golden standard for multireference systems. MRCI is routinely applied for the calculation of Potential Energy Surfaces (PESs) involving up to three or four atoms in several electronic states (see for example Refs. 54-59). For larger systems, the use of MRCI is not common due to its computational cost, and the complexity in some cases to achieve good convergence. However, if we can select a stable active space adapted to the description of the process under study, it becomes a powerful tool for the understanding of chemical reactivity.

The goal of this article is to understand the detailed mechanism of the spin-forbidden reactions between $\mathrm{O}_{2}$ and an organic substrate that do not require the presence of any co-factor. To address this question, we studied the reaction between DPA-CoA and $\mathrm{O}_{2}$ using two sets of quantum calculations: high level multireference $a b$-initio methods on a model system, and a DFT based QM/MM approach.

\section{RESULTS AND DISCUSSION}

As stated above, we are going to combine the results obtained using two kind of calculations. The first group consisted of MRCI calculations describing the 9 electronic states that are relevant for $\mathrm{O}_{2}$ addition to the 


\section{S-Methyl-but-3-enethioate model}<smiles>C=C[C@H](O)C(=O)SC</smiles>
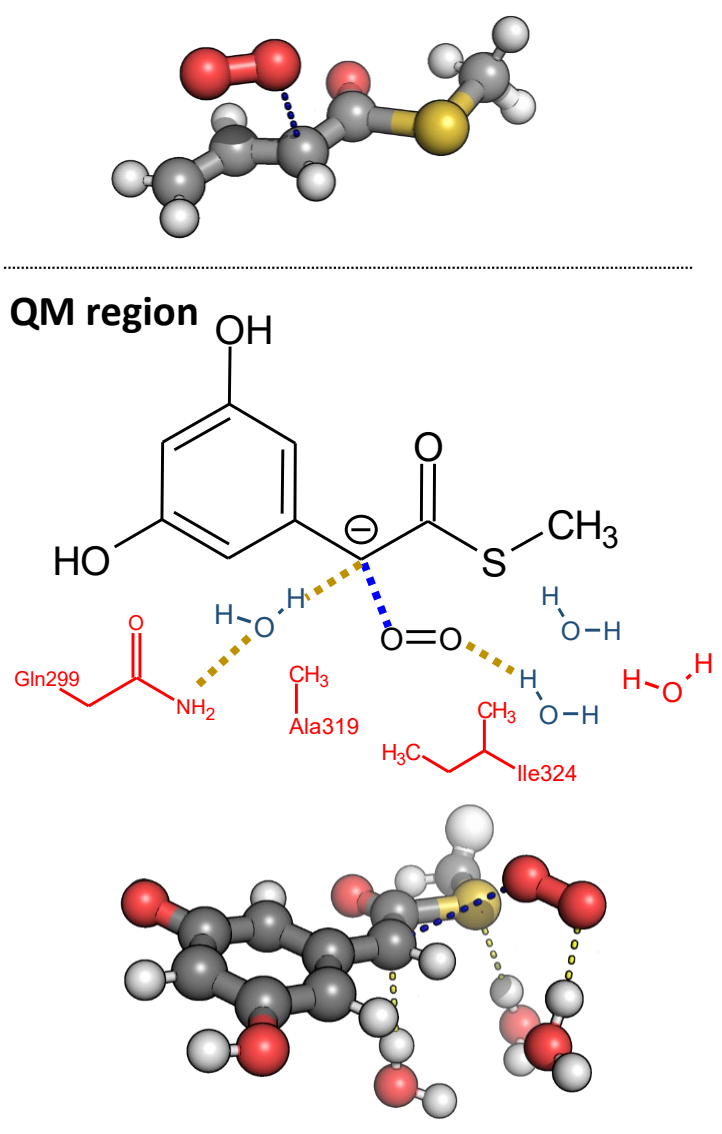

FIG. 2. Schematic representation of the atoms included in the S-Methyl-but-3-enethioate (top panel), and in the QM region for the QM/MM calculations (bottom panel). Two different QM regions were used in the latter calculations. A larger one including all residues highlighted, and a smaller one in which the residues highlighted in red were included in the MM region. The hydrogen bonds between DPA and the water molecules nearby are highlighted in yellow, while the distance between $\mathrm{C}_{\alpha}$ and $\mathrm{O}_{2}$ is highlighted in blue. For the size of clarity, we only show in the $3 \mathrm{D}$ representation the atoms included in the small QM region.

enolate. Due to the large computational cost of MRCI calculations, we used the model system depicted in the top panel of Fig. 2, where DPA-CoA has been replaced by S-Methyl-but-3-enethioate (hereinafter butenthioate). The second group of calculations consisted on a QM/MM description of the enzymatic reaction, relying on DFT methods. The atoms included in the QM-region (shown in the bottom panel of Fig. 2) are $\mathrm{O}_{2}$, the DPA moiety of the substrate, and the sidechain of three residues: Ala319 and Ile324 in the hydrophic pocket of $\mathrm{O}_{2}$, and Gln299. We also added to the QM region four water molecules nearby that could be involved either in the protonation of the peroxide formed or in the stabilization of DPACoA. The QM/MM calculations will provide clues on the role played by the protein scaffold to promote the process, and in particular will serve to discuss the relevance of the possible proton transfer steps.

\section{A. Reaction between $\mathrm{O}_{2}$ and S-Methyl-but-3-enethioate}

In its ground state, the electronic configuration of $\mathrm{O}_{2}$ is $\left(1 \sigma_{g}\right)^{2}\left(1 \sigma_{u}^{*}\right)^{2}\left(2 \sigma_{g}\right)^{2}\left(2 \sigma_{u}^{*}\right)^{2}\left(3 \sigma_{g}\right)^{2}\left(1 \pi_{u}\right)^{4}\left(1 \pi_{g}^{*}\right)^{2}$. The distribution of two electrons in two degenerated $\pi$ orbitals leads to 3 electronic states relatively close in energy: the ground ${ }^{3} \Sigma_{g}^{-}$state, the ${ }^{1} \Delta_{g}$ (doubly-degenerate, which splits into two states in the presence of any colliding partner), and the ${ }^{1} \Sigma_{g}^{+}$. The energy difference between ${ }^{1} \Sigma_{g}^{+}$and ${ }^{3} \Sigma_{g}^{-}$is only of $37.7 \mathrm{kcal} / \mathrm{mol}^{60}$, with ${ }^{1} \Delta_{g}$ lying between them $\left(22.64 \mathrm{kcal} / \mathrm{mol}\right.$ above $\left.{ }^{3} \Sigma_{g}^{-}\right)$. Further excited states involve excitation of one electron from $\left(1 \pi_{g}^{*}\right)$ to $\left(3 \sigma_{u}^{*}\right)$ orbital, but they appear much higher in energy and are unlikely to play any role in the intersystem crossing. On the other hand, butenthioate is a closed-shell molecule that does not present any excited state close in energy.

For the MRCI calculations we selected an active space that includes 4 electrons in 3 orbitals: the HOMO orbital of butenthioate (hereinafter $p_{C \alpha}$, although it is delocalized over the $3 s p^{2} \mathrm{C}$ atoms) and the two $1 \pi_{g}^{*}$ orbitals of $\mathrm{O}_{2}$, which are singly occupied in the ground state of $\mathrm{O}_{2}$ and that we will denote as $\pi_{\mathrm{O}_{2}}^{*}$. With this active space we can describe up to 9 electronic states: three triplets and six singlet states, that we will denote as $1-3{ }^{3}\left[\mathrm{DPA}-\mathrm{O}_{2}\right]$ and 1- ${ }^{1}\left[\mathrm{DPA}-\mathrm{O}_{2}\right]$ throughout the manuscript.

In Fig. 3 we show the energy profiles of the considered electronic states during the addition of $\mathrm{O}_{2}$ to butenthioate as a function of $r_{C_{\alpha} O}$, the distance between $\mathrm{C}_{\alpha}$ and $\mathrm{O}_{2}$ (the two atoms between which the new bond will be formed, as shown in Fig. 2). To build these energy profiles, optimized geometries were considered for each value of $r_{C_{\alpha} O}$, following the procedure described in the Methods section. It is worth noticing that, based on $\mathrm{QM} / \mathrm{MM}$ optimizations of the peroxide, we applied constraints to keep all the $\mathrm{C}$ atoms of butenthioate in the same plane. This constraint is only relevant at small $r_{C_{\alpha} O}$, where the peroxide is formed, and was chosen to better mimic the behavior of DPA-CoA in the protein environment (see Methods section for details).

We will first focus on the behavior displayed at large $r_{C_{\alpha} O}$ distances, where it is safe to assume that there is almost no interaction between butenthioate and $\mathrm{O}_{2}$, and the active-space orbitals do not mix. Under these circumstances, it is possible to correlate the adiabatic 

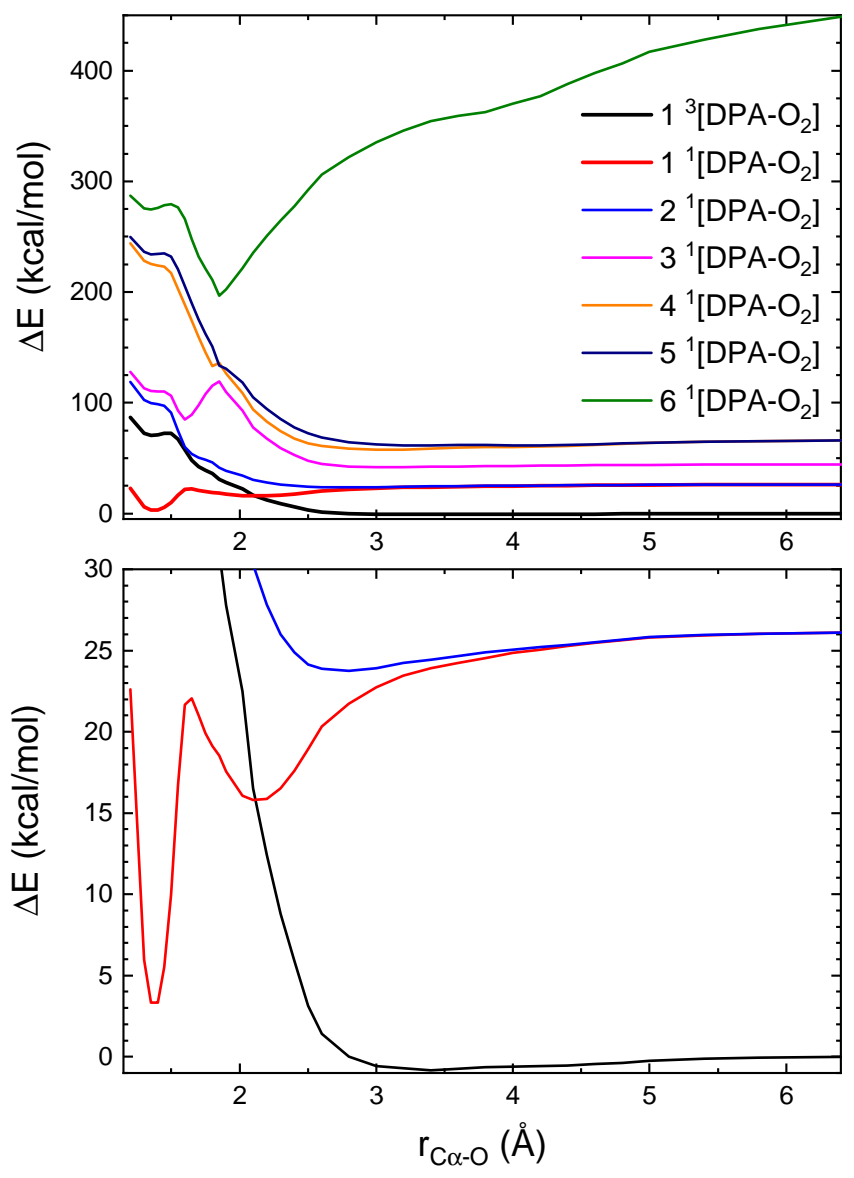

FIG. 3. Top panel: Energy profiles for the addition of $\mathrm{O}_{2}$ to S-Methyl-but-3-enethioate as a function of the $\mathrm{C}_{\alpha}-\mathrm{O}$ distance. The ground triplet state and the six singlets involved are shown. A zoom of the lower energy region is shown in the bottom panel. The two excited triplet states are not depicted for the sake of clarity.

states with the electronic configurations of the two colliding partners. The first 4 states (the ground triplet state, $1^{3}\left[D P A-O_{2}\right]$, and the three first singlet states, $\left.1-3{ }^{1}\left[D P A-O_{2}\right]\right)$ are well described with a $\left(p_{C \alpha}\right)^{2}\left(\pi_{\mathrm{O}_{2}}^{*}\right)^{2}$ electronic configuration, namely to a $\mathrm{DPA}^{-}-\mathrm{O}_{2}$ conformation, where the negative charge is located in the butenthioate. In this conformation, the 4 states correlate to the 4 lowest energy states of $\mathrm{O}_{2}$ : the ground ${ }^{3} \Sigma_{g}^{-}$state, the ${ }^{1} \Delta_{g}$ (doubly-degenerate), and the ${ }^{1} \Sigma_{g}^{+}$. In our calculations, the energy difference between ${ }^{1} \Delta_{g}$ and ${ }^{3} \Sigma_{g}^{-}$is $26.1 \mathrm{kcal} / \mathrm{mol}$, just a $15 \%$ larger than that observed for gas phase isolated $\mathrm{O}_{2}{ }^{60}$, and the same trend is observed for the difference between ${ }^{1} \Sigma_{g}^{+}$and ${ }^{3} \Sigma_{g}^{-}$, which confirms the validity of the theoretical methods applied.

The following 4 states, $4-5{ }^{1}\left[\mathrm{DPA}-\mathrm{O}_{2}\right]$ and $2-3$ ${ }^{3}\left[\mathrm{DPA}-\mathrm{O}_{2}\right]$ can be described using a $\left(p_{\mathrm{C \alpha}}\right)^{1}\left(\pi_{\mathrm{O}_{2}}^{*}\right)^{3}$ electronic configuration, namely DPA- $\mathrm{O}_{2}^{-}$, which corresponds to a radical-pair between the superoxo anion $\mathrm{O}_{2}^{-}$ and the butenthionyl radical. As there is no interaction between butenthioate and $\mathrm{O}_{2}$, all of them are de- generate at large $r_{C_{\alpha} O}$. The energy difference between 1 ${ }^{3}\left[\mathrm{DPA}-\mathrm{O}_{2}\right]$ and these states is $66.1 \mathrm{kcal} / \mathrm{mol}$, and corresponds to the energy required to transfer one electron from butenthioate to $\mathrm{O}_{2}$ in gas phase. The presence of the protein environment stabilizes the charge on the $\mathrm{O}_{2}^{-}$ shifting this energy to $36.6 \mathrm{kcal} / \mathrm{mol}$. This value, however, is significantly larger than that calculated for the reaction between $\mathrm{O}_{2}$ and flavin, $2.8 \mathrm{kcal} / \mathrm{mol}$ at a DFT level (and implicit water solvent). ${ }^{30}$ This difference may explain why enzymes use flavin as a redox catalyzer.

The electronic configuration of the last state, 6 ${ }^{1}\left[\mathrm{DPA}-\mathrm{O}_{2}\right]$, is $\left(p_{\mathrm{C \alpha}}\right)^{0}\left(\pi_{\mathrm{O}_{2}}^{*}\right)^{4}$ or $\mathrm{DPA}^{+}-\mathrm{O}_{2}^{2-}$. It corresponds to the interaction between the peroxo anion $\left(\mathrm{O}_{2}^{2-}\right)$ and the butenthionyl cation. The energy of this state is very high (more than $450 \mathrm{kcal} / \mathrm{mol}$ ), as it requires the transference of two electrons from butenthioate to $\mathrm{O}_{2}$. Its energy, however, decreases quickly with the $r_{C_{\alpha} O}$ distance, as expected for an ion-ion interaction $\left(E \propto r^{-1}\right)$. If we had used a larger active space, we would have obtained several states between $5^{1}\left[\mathrm{DPA}-\mathrm{O}_{2}\right]$ and 6 ${ }^{1}\left[\mathrm{DPA}-\mathrm{O}_{2}\right]$, and this would have impaired the description of the $6{ }^{1}\left[\mathrm{DPA}-\mathrm{O}_{2}\right]$. In spite of the very large energy difference between $6{ }^{1}\left[\mathrm{DPA}-\mathrm{O}_{2}\right]$ and the ground $1^{3}\left[\mathrm{DPA}-\mathrm{O}_{2}\right]$ state, the former state will be important to describe the formation of the peroxide, as we will discuss below.

When $\mathrm{O}_{2}$ and butenthioate approach each other, the orbitals mix and the correspondence between our adiabatic states and the electronic configuration of the two independent molecules is not longer valid. At $r_{C_{\alpha} O}<4$ $\AA$, the interaction between $\mathrm{O}_{2}$ and butenthioate is already strong enough to break the degeneracy of 2-3 ${ }^{1}\left[D P A-O_{2}\right]$ (former ${ }^{1} \Delta_{g}$ states). The inspection of the active space orbitals reveals that $p_{C \alpha}$ starts to overlap with one of the two no longer equivalent $\pi_{\mathrm{O}_{2}}^{*}$. The same effect is observed for the four DPA- $\mathrm{O}_{2}^{-}$states, also no longer degenerate. The interaction of butenthioate and $\mathrm{O}_{2}$ makes that for $2 \AA<r_{C_{\alpha} O}<3 \AA$ only $1^{1}\left[\mathrm{DPA}-\mathrm{O}_{2}\right]$ and $6^{1}\left[\mathrm{DPA}-\mathrm{O}_{2}\right]$ are not repulsive. For $r_{C_{\alpha} O}<2 \AA$, the ground singlet state becomes more stable than the triplet state, showing a minimum for $r_{C_{\alpha} O}=$ $1.4 \AA$. It is also worth mentioning that the energy of $6^{1}\left[\mathrm{DPA}-\mathrm{O}_{2}\right]$ reaches a minimum at $1.85 \AA$, after which it rises. This behaviour is not compatible with the description of an ion-ion interaction, so it suggests that this state has changed its character due to an avoided crossing caused by strong non-adiabatic couplings with other singlet states.

In the bottom panel of Fig. 3 we show an expanded view that allows us to appreciate the main features of the PES for the three lowest energy states, $1^{3}\left[\mathrm{DPA}-\mathrm{O}_{2}\right]$ and $1-2^{1}\left[\mathrm{DPA}-\mathrm{O}_{2}\right]$. The $1^{3}\left[\mathrm{DPA}-\mathrm{O}_{2}\right]$ state is purely repulsive, and its energy rises quickly at $r_{C_{\alpha} O}<2.5 \AA$. That behavior is shared with the $2^{1}\left[\mathrm{DPA}-\mathrm{O}_{2}\right]$ state. On the contrary, $1^{1}\left[\mathrm{DPA}-\mathrm{O}_{2}\right]$ is attractive, and displays a double-well structure. The energy of the minimum of the first well is $15.9 \mathrm{kcal} / \mathrm{mol}$ at $r_{C_{\alpha} O}=2.1 \AA$, a distance large enough so the $\mathrm{C}_{\alpha} \mathrm{O}$ covalent bond is still not 

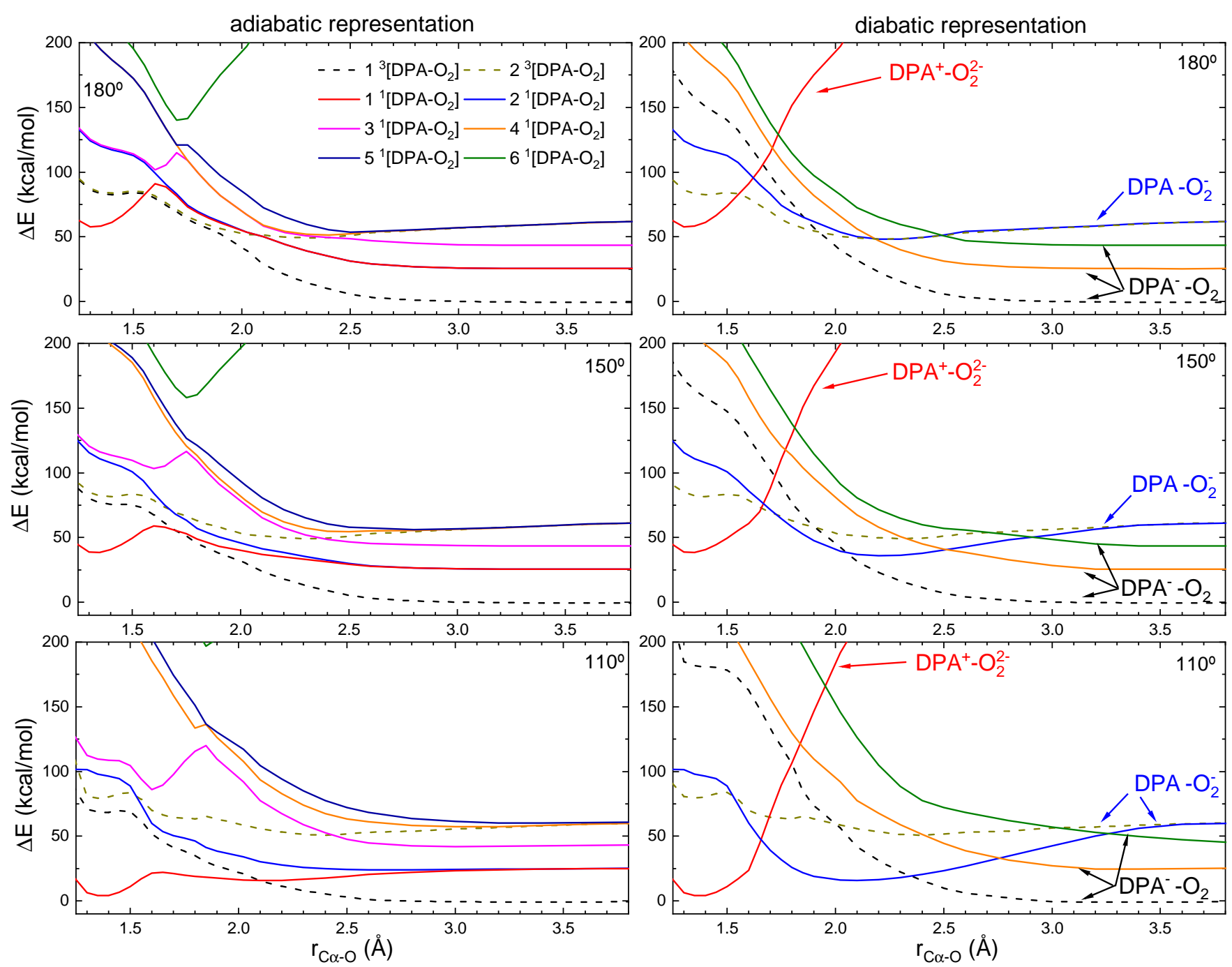

FIG. 4. Energy profiles for the addition of $\mathrm{O}_{2}$ to S-Methyl-but-3-enethioate as a function of the $\mathrm{C}_{\alpha}-\mathrm{O}$ distance and the $\mathrm{C}_{\alpha}$-O-O angle. In the left panels the adiabatic curves are shown and their corresponding diabatic curves are shown in the right panels. $\widehat{C O O}=110^{\circ}$ corresponds to the minimum energy at the MECP shown in Fig. 3. The curves for the singlet (triplet) states are shown in solid (dashed) lines.

formed. The electronic wavefunction in this well exhibits a strong superoxo $\left(\mathrm{O}_{2}^{-}\right)$character and seems to arise from a strong charge-dipole interaction which allows to stabilize significantly this state. Interestingly, the singlet and the triplet states cross at the bottom of this well, which coincides to the MECP. The second minimum is significantly deeper (just $3.4 \mathrm{kcal} / \mathrm{mol}$ higher than the minimum of the triplet state) and is located at $r_{C_{\alpha} O}=1.4$ $\AA$, this well is associated to the peroxide.

The two minima are separated by a maximum on the one dimension curve, which corresponds to a saddlepoint. It lies slightly below the asymptotic energy of the $1^{1}\left[\mathrm{DPA}-\mathrm{O}_{2}\right]$ state. This saddle-point is associated to the barrier of the superoxo to peroxo transformation, and arises from an avoided crossing. The huge gap in energy between states at the saddle-point indicates that the non-adiabatic couplings are very strong in this re- gion, suggesting that the charge transfer may occur adiabatically. We should point out that the barrier height predicted by our method is probably overestimated, partially because the constraints imposed to keep the structure planar and also, as we will discuss later, due to the small size of the active space. The planar restraint imposed to butenthioate also affects the relative energy of the minimum of the first singlet and triplet states, and causes the $1^{1}\left[\mathrm{DPA}-\mathrm{O}_{2}\right]$ minimum to lie above the minimum energy $1^{3}\left[\mathrm{DPA}-\mathrm{O}_{2}\right]$ state. It should be noticed that this does not affect the energy profile for $r_{C_{\alpha} O}>$ $2 \AA$, where the peroxide bond is not formed, and the molecule is intrinsically planar. Thus, the MECP region should not be affected by the planar restraint.

To get more insight onto the nature of the states that are involved in the spin-orbit process, we generated the quasi-diabatic states for the addition of $\mathrm{O}_{2}$ to buten- 
thioate. The quasi-diabatic states have the property that they retain the character displayed at asymptotic distances, so using this approximation it is possible to extend the assignment of an electronic configuration for independent $\mathrm{O}_{2}$ and butenthioate to small $\mathrm{C}_{\alpha}-\mathrm{O}$ distances. In order to ascertain the strength of the non-adiabatic couplings (and therefore their effects on the potential energy curves), we calculated the diabatic states as a function of $r_{C_{\alpha} O}$ for three different $\mathrm{C}_{\alpha}-\mathrm{O}-\mathrm{O}$ angles: $110^{\circ}$, which is close to the value obtained for the fully relaxed geometries shown in Fig. $3,180^{\circ}$ for which $\mathrm{O}_{2}$ inserts perpendicular to the butenthioate plane and minimizes the overlap between $\left(p_{C \alpha}\right)$ and $\left(\pi_{\mathrm{O}_{2}}^{*}\right)$ orbitals, and $150^{\circ}$, an intemediate value in order to see the evolution. In the left panels of Fig. 4 we show the adiabatic curves of the approaching of $\mathrm{O}_{2}$ to butenthioate, while the diabatic states are shown in the right panels.

In the absence of overlap between $\left(p_{C \alpha}\right)$ and $\left(\pi_{\mathrm{O}_{2}}^{*}\right)$ orbitals, $\widehat{C_{\alpha} O O}=180^{\circ}$, only the $6^{1}\left[\mathrm{DPA}-\mathrm{O}_{2}\right]$ adiabatic state is not repulsive for $r_{C_{\alpha} O}>2 \AA$. Since the nonadiabatic couplings are small for this orientation, the diabatic and adiabatic representations are very similar. Interestingly, at $\widehat{C_{\alpha} O O}=180^{\circ}$, the $1-2^{1}\left[\mathrm{DPA}-\mathrm{O}_{2}\right]$ as well as $4-5^{1}\left[\mathrm{DPA}-\mathrm{O}_{2}\right]$ adiabatic states are nearly degenerate for distances down to $1.7 \AA$. For this orientation and for $r_{C_{\alpha} O}=1.4 \AA$, we observe a shallow minimum on the first singlet state. The quasi-diabatization procedure reveals unambiguously that this minimum correlates to the $6^{1}\left[\mathrm{DPA}-\mathrm{O}_{2}\right]$ ionic state, and it is associated to a $\mathrm{DPA}^{+}$$\mathrm{O}_{2}^{2-}$ (peroxo) conformation.

When the orientation deviates from $\widehat{C_{\alpha} O O}=180^{\circ}$, $\left(p_{C \alpha}\right)$ and $\left(\pi_{\mathrm{O}_{2}}^{*}\right)$ orbitals mix, non-adiabatic couplings get stronger and the degeneracy between $1-2^{1}\left[\mathrm{DPA}-\mathrm{O}_{2}\right]$ and between $4-5^{1}\left[\mathrm{DPA}-\mathrm{O}_{2}\right]$ breaks again. It is interesting to note here that with the increasing strength of the non-adiabatic couplings, the adiabatic picture deviates progressively from the diabatic view, implying that couplings may affect several states simultaneously. Still, applying the change to the diabatic representation, we can establish that the peroxide minimum is consistently associated to $\mathrm{DPA}^{+}-\mathrm{O}_{2}^{2-}$, as expected. The diabatization also permits to assign the first minimum of the first singlet state, which coincides to the MECP, to the well associated to a DPA-O ${ }_{2}^{-}$state. This finding is confirmed by the coefficients of the CI vector displayed in Fig. S1.

The mechanism that emerges from these results is the following: $\mathrm{O}_{2}$ addition starts with a first charge-transfer between butenthioate and $\mathrm{O}_{2}$, leading to the formation of a radical pair with the formation of the superoxo anion. This charge-transfer is concomitant with the intersystem crossing, and is therefore mediated by the strength of the SOC in this region. Following this process, a second charge-transfer step takes place, in this case between two singlet states and leads to the formation of the peroxide. The deep well associated to the minimum of the diabatic superoxo state $\left(\mathrm{DPA}-\mathrm{O}_{2}^{-}\right)$is then responsible of lowering the energy of the MECP, and accordingly to the promo-

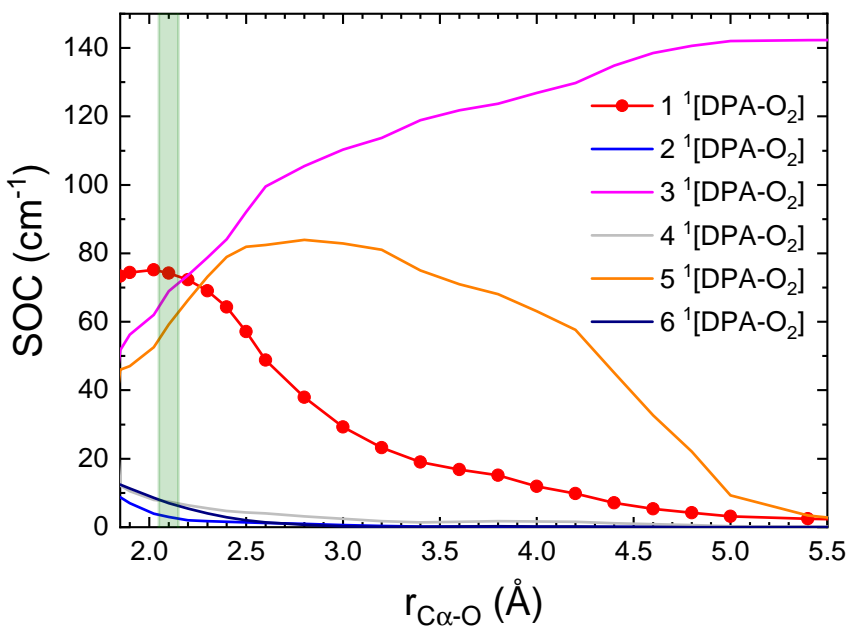

FIG. 5. Values of the Spin-Orbit Coupling (SOC) between the ground triplet state and the six singlets involved in the oxidation of DPA-CoA as a function of the $\mathrm{C}_{\alpha}-\mathrm{O}$ distance. The values of the SOC between the ground singlet and triplet states are shown as red-closed circles. The region in which the MECP was found is shaded in green.

tion of the spin flipping.

In the afore discussion we have assumed that electron is transferred "at once". However, this classical picture is not fully correct because, although the dominant character in the well is the one of the superoxo, both DPA-O ${ }_{2}^{-}$ and $\mathrm{DPA}^{-}-\mathrm{O}_{2}$ states are coupled and strongly mixed. It is therefore more accurate to discuss about transference of electronic density. To account for this effect, in Fig. $\mathrm{S} 2$ we show the evolution of the partial charge that is accumulated on the $\mathrm{O}_{2}$ moiety as a function of $r_{C_{\alpha} O}$. As can be observed, spin flipping is associated with an appreciable electron density transfer from buthentioate to $\mathrm{O}_{2}$, although part of the electronic density had been already been transferred to the $\mathrm{O}_{2}$ before the intersystem crossing.

The nature of the two states involved in the spinchange process has important implications for the feasibility of the process. According to El-Sayed rules, if the change of spin is associated with a change in the orbital angular momentum (or electronic configuration), the spin orbit-coupling would be strong, and the rate coefficient of the spin-forbidden process will be large ${ }^{61}$ even if there is only a small change in electronic density. ${ }^{62}$ The values of the SOC between the ground triplet state and the six singlet states are shown in Fig. 5. At large $r_{C_{\alpha} O}$ distances, only the SOC with the $3^{1}\left[\mathrm{DPA}-\mathrm{O}_{2}\right]\left({ }^{1} \Sigma_{g}^{+}\right.$ of $\mathrm{O}_{2}$ ) is large, as it was observed for isolated $\mathrm{O}_{2} \cdot{ }^{63,64}$ When $\mathrm{O}_{2}$ and butenthioate approach, the value of the SOC between the first singlet and triplet states increases smoothly with the energy, which could be explained by the transfer of electronic density from butenthioate to $\mathrm{O}_{2}$ (and the higher weight of the $\mathrm{O}_{2}^{-}$states in the CI vector of the first singlet state, as it was shown in Fig. S1). The SOC reaches a plateau value of $75 \mathrm{~cm}^{-1}$ in the region of 


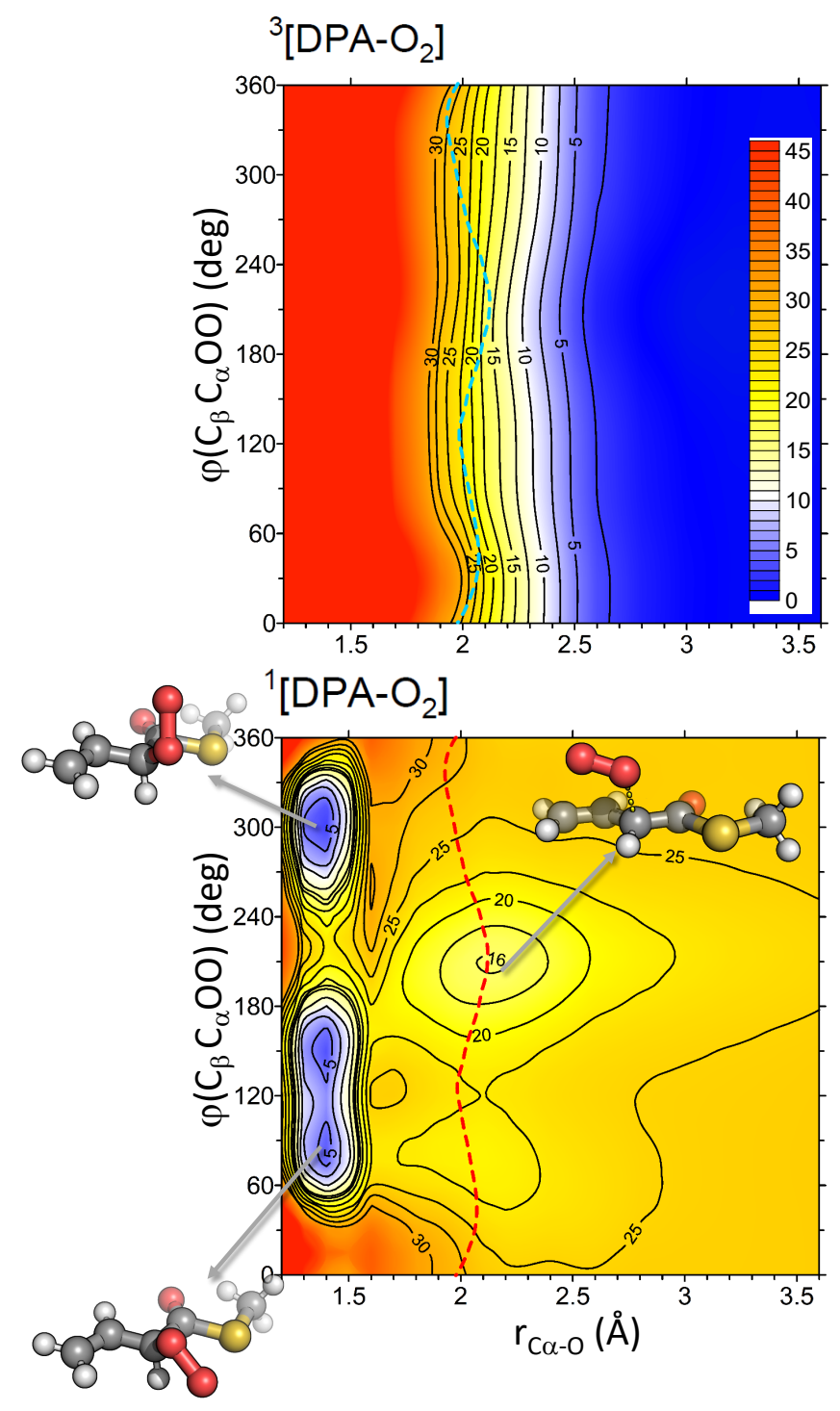

FIG. 6. 3D contour map of the energy profile for the addition of $\mathrm{O}_{2}$ to S-Methyl-but-3-enethioate as a function of $\mathrm{C}_{\alpha}-\mathrm{O}$ distance and the $\mathrm{C}_{\beta}-\mathrm{C}_{\alpha}-\mathrm{O}-\mathrm{O}$ dihedral angle. Top panel: Lowest triplet state. Bottom panel: Lowest singlet state. The crossing seam is shown as a dashed line on top of the contour maps. The geometry of the MECP and two of the peroxide minima are shown. Energies are in $\mathrm{kcal} / \mathrm{mol}$.

the MECP, in accordance with the value for $76.5 \mathrm{~cm}^{-1}$ estimated based on $\mathrm{O}_{2}^{-}$spectroscopic constant. ${ }^{31,33}$ This relatively strong $\mathrm{SOC}$ is enough to promote the intersystem crossing, even in the absence of any metal cofactor.

Larger SOC values are typically associated to the presence of heavy atoms, so to check if the presence of the $\mathrm{S}-\mathrm{CH}_{3}$ group could play an important role, we calculated the SOC replacing $\mathrm{S}$ by $\mathrm{O}$, obtaining similar values of the SOC. Hence, we can conclude that $\mathrm{S}$ does not influence the SOC, and that the SOC obtained should be similar for other co-factorless spin-forbidden reactions between $\mathrm{O}_{2}$ and enolates.

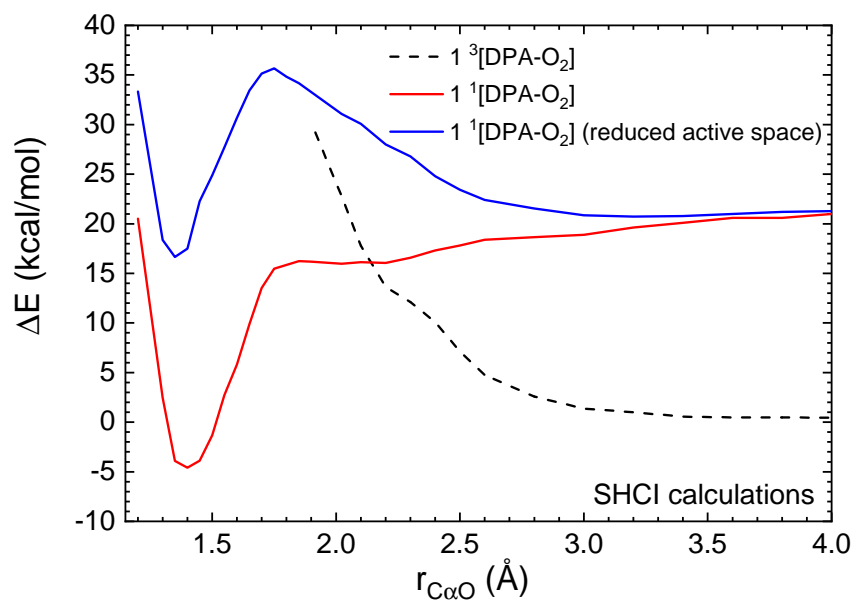

FIG. 7. Energy profile for the addition of $\mathrm{O}_{2}$ to S-Methyl-but3-enethioate as a function of the $\mathrm{C}_{\alpha}$-O distance using SHCI method. The solid blue curve shows the results obtained for the singlet state when the $3 \mathrm{~d}$-orbitals of $\mathrm{O}_{2}$ have been excluded from the active site.

To get more insight about the geometry of the crossing seam (the region in which singlet and triplet states are degenerate), in Fig. 6 we show the energy surface cuts of the approach of $\mathrm{O}_{2}$ to butenthioate as a function of both $r_{C_{\alpha} O}$ and $\varphi\left(\mathrm{C}_{\beta}-\mathrm{C}_{\alpha} \mathrm{OO}\right)$, the dihedral angle between $\mathrm{C}_{\beta}$ - $\mathrm{C}_{\alpha}$-O-O for the first singlet and triplet states $\left(\mathrm{C}_{\beta}\right.$ was defined in Fig. 2). For the triplet state, the energy barely depends on $\varphi\left(\mathrm{C}_{\beta}-\mathrm{C}_{\alpha} \mathrm{OO}\right)$, and the curve is purely repulsive (as it was expected from Fig. 3). On the other hand, the PES of the first singlet state is considerably anisotropic. The first well, that associated to DPA- $\mathrm{O}_{2}^{-}$, is located at $r_{C_{\alpha} O}=2.1 \AA$, and $\varphi\left(\mathrm{C}_{\beta}-\mathrm{C}_{\alpha} \mathrm{OO}\right) \approx 210^{\circ}$. A second shallower minimum is found at $\varphi\left(\mathrm{C}_{\beta}-\mathrm{C}_{\alpha} \mathrm{OO}\right) \approx$ $60^{\circ}$, when the $\mathrm{O}_{2}$ is closer to $\mathrm{S}$ than to the double bond. The absolute minimum, when the peroxide is formed and associated to the $\mathrm{DPA}^{+}-\mathrm{O}_{2}^{2-}$ configuration, is reached at $r_{C_{\alpha} O}=1.4 \AA$, and three different values of $\varphi\left(\mathrm{C}_{\beta^{-}}\right.$ $\mathrm{C}_{\alpha} \mathrm{OO}$ ). The crossing seam is shown as a dashed line on top of the contour maps. Regardless of the value of $\varphi\left(\mathrm{C}_{\beta}-\mathrm{C}_{\alpha} \mathrm{OO}\right)$, the seam is found for $2 \AA<r_{C_{\alpha} O}<2.1$ $\AA$, where the triplet PES cuts the singlet PES at the DPA-O $\mathrm{O}_{2}^{-}$minimum.

The geometry of the MECP, and two of the absolute minima are also depicted in Fig. 6. The carbon scaffold of butenthioate was restrained to be planar and, at the MECP, the bond between $\mathrm{H}$ and $\mathrm{C}_{\alpha}$ nearly lies in the butenthioate plane, suggesting that $\mathrm{C}_{\alpha}$ still presents a $\mathrm{sp}^{2}$ hibridation, and that the aromatic cloud is not yet perturbed by the $\mathrm{O}_{2}$ approach. The O-O distance is 1.29 $\AA$, closer to that observed for $\mathrm{O}_{2}^{-}(1.345 \AA)$ than for the singlet state of $\mathrm{O}_{2}(1.215 \AA) \cdot{ }^{65}$ At smaller $r_{C_{\alpha} O}$, the hydrogen progressively folds out of the plane while the $\mathrm{C}_{\alpha} \mathrm{O}$ bond is formed, as expected for a $\mathrm{sp}^{3}$ hibridation of $\mathrm{C}_{\alpha}$.

To study the effect that the inclusion of more orbitals in the active space may have in the results, we 


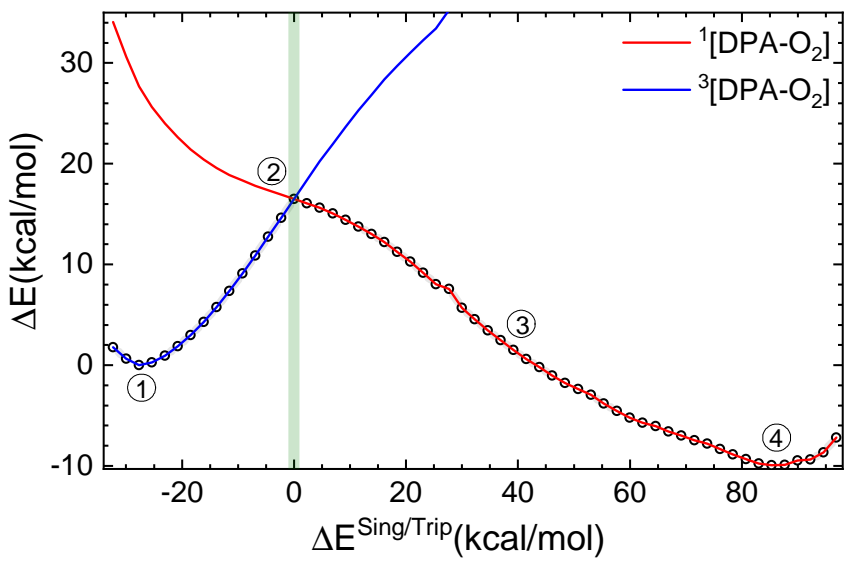

FIG. 8. QM/MM energy profile for the addition of $\mathrm{O}_{2}$ to the DPA model as a function of the energy difference between the ground singlet and triplet states. $\Delta \mathrm{E}^{\mathrm{Sing} / \text { Trip }}=0$ corresponds to the MECP, and it is shaded in green. The framed numbers highlight the values of $\Delta \mathrm{E}^{\text {Sing/Trip }}$ corresponding to the structures shown in Fig. 9

recalculated the energy profile for the addition of $\mathrm{O}_{2}$ to butenethioate using the Semistochastic Heat-Bath Configuration Interaction (SHCI) method, ${ }^{66-68}$ a recently developed semistochastic method that permits to circumvent the limitation in the number of orbitals of the active space, and can account for dynamic correlations via perturbation theory. For this SHCI calculations, the active space was formed by 23 orbitals, including the $\mathrm{p}_{z}$ orbitals of the $\mathrm{sp}^{2} \mathrm{C}$ and the $2 \mathrm{p}$ and $3 \mathrm{~d}$ orbitals of $\mathrm{O}_{2}$ (the 23 orbitals are shown in Fig. S3). The results using SHCI are shown in Fig. 7 and are in qualitative agreement with the MRCI calculations, being the main differences the much lower barrier between the superoxo and peroxo region, and the larger stability of the peroxide. The height of the MECP, however, is very similar to that obtained using MRCI. In Fig. 7 we also show the SHCI results obtained when the $3 \mathrm{~d}$ orbitals of $\mathrm{O}_{2}$ were not included. Interestingly, in this case there is a huge barrier that prevents the intersystem crossing. It clearly shows that the SHCI results strongly depend on the number of orbitals of the active space, which let us believe that the MRCI barrier between peroxo and superoxo would be significantly smaller if more orbitals were added to the active space.

\section{B. Reaction between $\mathrm{O}_{2}$ and DPA-CoA in DpgC}

Once we have described the nature of the electronic states involved in the process, and how intersystem crossing could take place in our model system, we turn to the study of the addition of $\mathrm{O}_{2}$ to DPA-CoA catalyzed by DpgC, which is likely to be the rate limiting step for the formation of DPGX (Fig. 1). Due to the larger system size, we cannot go beyond DFT calculations. This impedes the calculation of all the relevant electronic states, but allow us to determine the role of the protein environment and to study the possible proton transfer.

To analyze the mechanism of this reaction, we carried out restrained geometry optimizations along the energy difference between the first singlet and triplet state ( $\Delta E^{\text {Sing/Trip }}$, see methods section for more details). The selection of $\Delta E^{\text {Sing/Trip }}$ as a reaction coordinate has two important advantages: i) the MECP is localized exactly at $\Delta E^{\text {Sing/Trip }}=0$, and ii) the evolution from the triplet to the singlet state is smoother.

The QM/MM energy profile for the $\mathrm{O}_{2}$ addition to DPA-CoA is shown in Fig. 8. QM/MM results show that both the triplet and the singlet states show only one minimum, very broad for the case of the singlet state. Interestingly, we observe that the MECP is the only barrier for the reaction, and accordingly should act as the dynamic bottleneck. MECP lies $16.7 \mathrm{kcal} / \mathrm{mol}$ above the minimum for the triplet state, in very good agreement with the value obtained for the $\mathrm{O}_{2}$-butenthioate model. Formation of the peroxide is driven by a exothermicity of $9.9 \mathrm{kcal} / \mathrm{mol}$.

To investigate the effect of the protein in the QM/MM energy profile, we repeated the calculations using a reduced QM region, in which Ile324, Gln299, Ala319, and one of the water molecules were excluded from the QM region. The obtained results were pretty similar, except for the exothermicity which is somewhat smaller (5.6 $\mathrm{kcal} / \mathrm{mol}$ ) evincing that the protein scaffold plays only a minor role in the promotion of the intersystem crossing besides the stabilization of the enolate (results do not shown).

The structures of the minimum of the triplet state, the MECP, the point at $\Delta E^{\text {Sing } / \text { Trip }}=39 \mathrm{kcal} / \mathrm{mol}$, and the minimum of the singlet state are depicted in Fig. 9. The structure obtained for the triplet state minimum is very similar to the crystal structure, although in this case $\mathrm{O}_{2}$ has moved slightly closer to $\mathrm{C}_{\alpha}$. Indeed, the position of the two water molecules that are close to $\mathrm{O}_{2}$ and $\mathrm{C}_{\alpha}$ is very similar in the crystal. Regarding these water molecules, it could have been expected that one of the water molecules could protonate the triplet $\mathrm{O}_{2}$ as it was predicted for the reaction between $\mathrm{O}_{2}$ and flavin. ${ }^{30,33,69}$ However, at the MECP, the proton is still on the water. In fact, the main differences between the minimum associated to the triplet and the MECP are that the hydrogen bound to $\mathrm{C}_{\alpha}$ has moved slightly below the plane formed by the $\pi$ system of DPA, and that $\mathrm{O}_{2}$ is significantly closer to $\mathrm{C}_{\alpha}$. This is shown in Fig. 10, where we display the evolution of the $\mathrm{C}_{\alpha}-\mathrm{O}$, and the $\mathrm{O}-\mathrm{O}$ distances along the reaction path. In the figure we also show the progress of the proton transfer, which is defined as the difference between the $r_{\mathrm{H}-\mathrm{OH}}$ and the $r_{\mathrm{O}-\mathrm{H}_{2} \mathrm{O}}$ (as defined in Fig. $9)$.

Between the triplet minimum and the MECP, $r_{C_{\alpha} O}$ has changed from 3.2 to $2 \AA$, in excellent agreement with the results obtained for the butenthioate model. Based on these results, the first triplet state is purely repulsive, so systems in which the MECP is found at larger (smaller) 

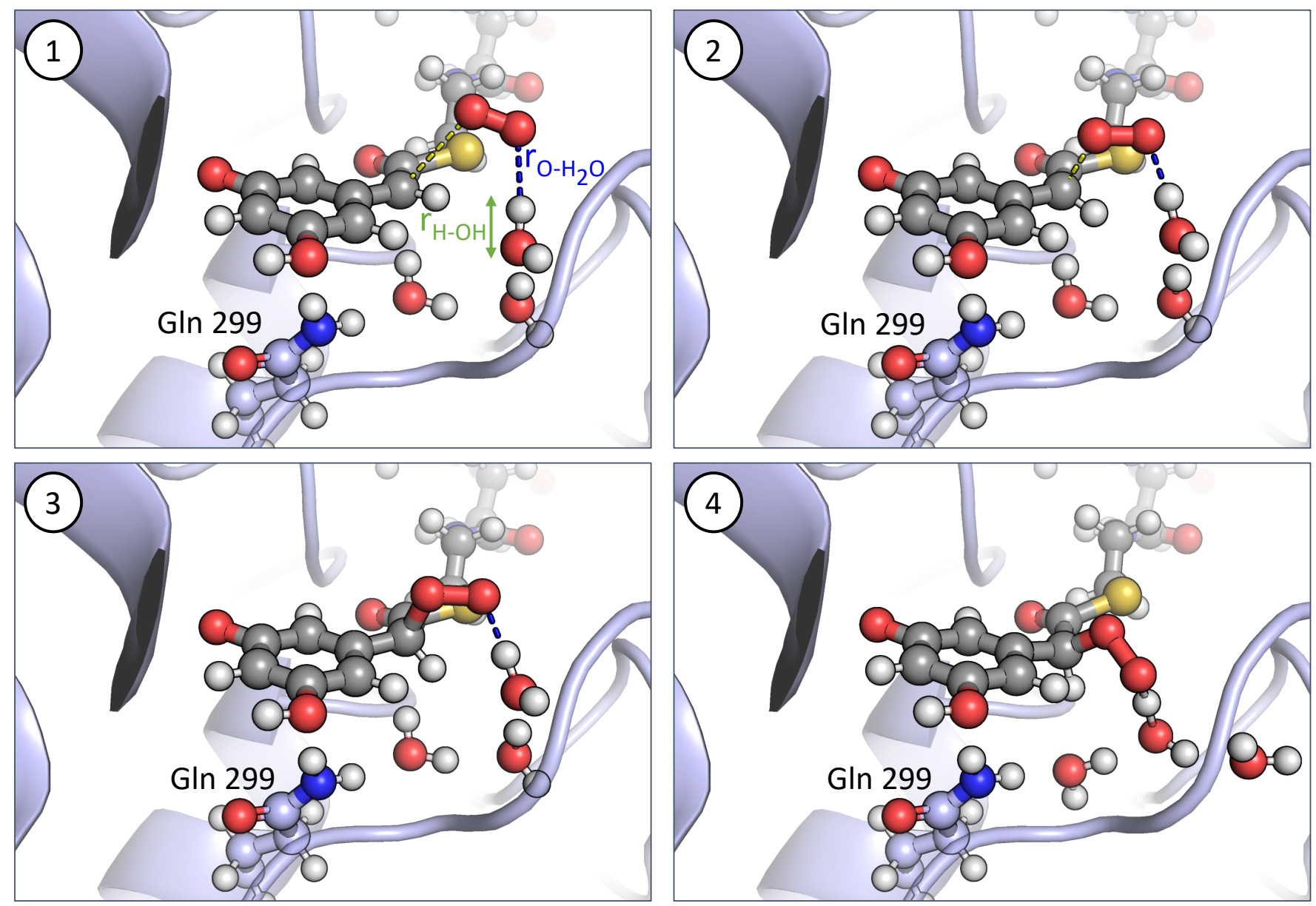

FIG. 9. QM/MM structures along the $\Delta \mathrm{E}^{\text {Sing/Trip }}$ path. 1: Structure corresponding to the optimized triplet geometry. 2: Structure of the system at the MECP. 3: Structure of the system for $\Delta \mathrm{E}^{\text {Sing/Trip }} \approx 40 \mathrm{kcal} / \mathrm{mol}$. 4: Structure of the optimized singlet structure. $\mathrm{C}_{\alpha}-\mathrm{O}, \mathrm{H}-\mathrm{OH}$, and $\mathrm{O}-\mathrm{H}_{2} \mathrm{O}$ distances are shown in yellow, green, and blue. Gln299, three water molecules that are included in the QM region, DPA-CoA, and $\mathrm{O}_{2}$ are shown atomistically.

$r_{C_{\alpha} O}$ are likely to be more (less) reactive.

After the MECP, $r_{C_{\alpha} O}$ decreases slowly, and only reaches its equilibrium value of $1.4 \AA$ for $\Delta E^{\text {Sing/Trip }}>$ $45 \mathrm{kcal} / \mathrm{mol}$. Interestingly, at $\Delta E^{\mathrm{Sing} / \text { Trip }}=39 \mathrm{kcal} / \mathrm{mol}$ the structure resembles that obtained for the MECP with the sole exception that the $\mathrm{C}_{\alpha}-\mathrm{O}$ bond is formed. Still, the proton has not been transferred to the peroxide. It means that for this reaction, the proton transfer occurs significantly after the peroxide is formed. Probably that is the reason why there are not basic residues around the active site that could donate a proton to $\mathrm{O}_{2}$ as it was observed for other cofactorless reactions. ${ }^{30,33,69}$ Proton transfer drifts the system away from the MECP and, even in the minimum associated to the singlet state, the proton is only shared between the peroxide and the water.

It is also worth mentioning that in the minimum of the singlet state, the water-wire that connects the bulk to the active site has reorganized. In particular, the water molecule that connects Gln299 and $\mathrm{C}_{\alpha}$ is reoriented to a position suitable for the extraction of the $\mathrm{C}_{\alpha}$ proton, which would facilitate the following step in DPGX formation. Interestingly, this water molecule was resolved in the crystal structure, and was not exchanged with the solvent throughout the MD simulations.

Combining the height of the MECP obtained in the QM/MM calculations, with the SOC obtained using our model, and plugging them in the Landau-Zenner equation ${ }^{45,50}$ we could estimate $\mathrm{k}_{\text {cat }}$. We obtained a hopping probability of 0.06 , which is equivalent to an increase of $1.6 \mathrm{kcal} / \mathrm{mol}$ in the activation energy at $300 \mathrm{~K}$. Using these values, we obtain a $\mathrm{k}_{\text {cat }}=0.23 \mathrm{~s}^{-1}$, very close to the experimental value of $0.15 \mathrm{~s}^{-1} .{ }^{43}$ The quantitative agreement between experiment and the simulation is coincidental, considering the approximations used. However, it supports the validity of the mechanism proposed in this article for the intersystem crossing and subsequent formation of the peroxide. 

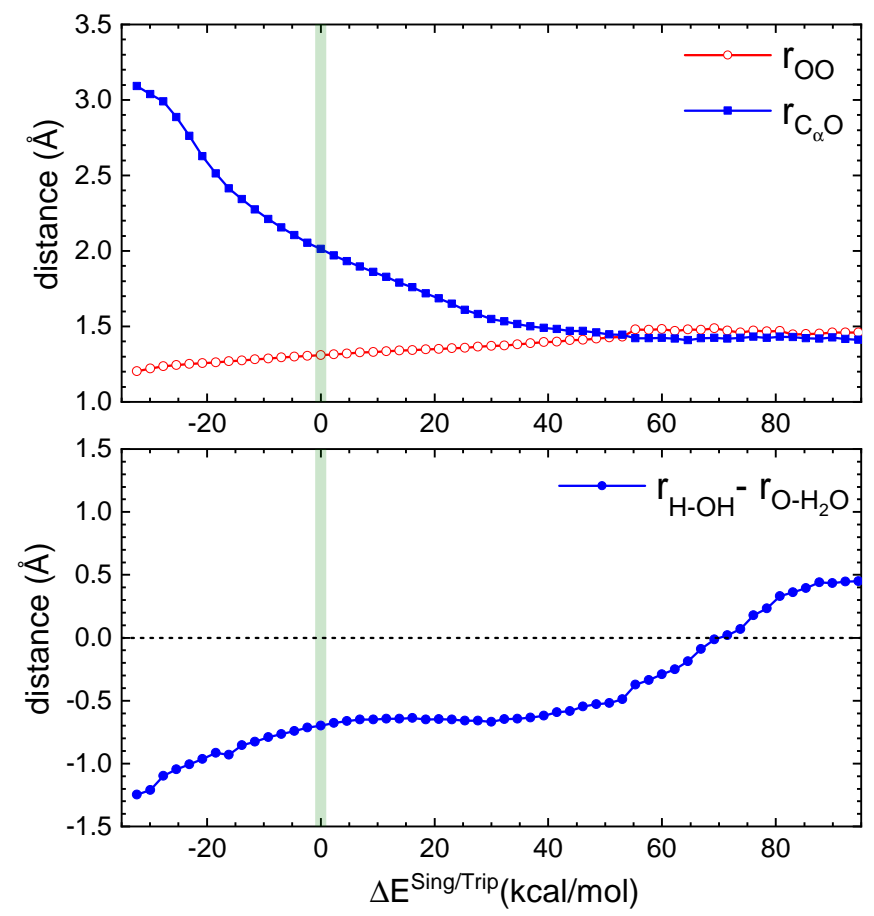

FIG. 10. Evolution of the O-O, $\mathrm{C}_{\alpha}-\mathrm{O}$, and proton transfer coordinate (defined as $r_{\mathrm{H}-\mathrm{OH}}-r_{\mathrm{O}-\mathrm{H}_{2} \mathrm{O}}$ ) along the $\Delta \mathrm{E}^{\text {Sing/Trip }}$ path. $\Delta \mathrm{E}^{\mathrm{Sing} / \text { Trip }}=0$ corresponds to the MECP, and it is shaded in green.

\section{CONCLUSIONS}

Incorporation of molecular oxygen into a organic substrate involves a change in the spin-state of the system, i.e. proceeds via intersystem crossing. To catalyze these reactions, enzymes typically require metal cofactors, generally iron. Intriguingly, a subgroup of oxygenases perform these reactions without the presence of any co-factor. Here, we have investigated the mechanism of this spin-forbidden process using MRCI quantum calculations and $\mathrm{QM} / \mathrm{MM}$ simulations on the reaction between DPA-CoA and $\mathrm{O}_{2}$ catalyzed by DpgC. Using MRCI calculations, we calculated the potential energy surfaces of the 9 concurrent electronic states on an equal footing, and assigned an electronic configuration to each of them via a diabatization procedure. Our results provide evidence that, once the enolate is formed, the reaction can occur without any previous activation of $\mathrm{O}_{2}$ and that successive electron transfer leads to the formation of peroxide. The minimum energy crossing point, which plays the role of an effective barrier for spin-forbidden processes, lies only $16.7 \mathrm{kcal} / \mathrm{mol}$ above the DPA-CoA $+{ }^{3} \mathrm{O}_{2}$ asymptote, which is compatible with experimental $\mathrm{k}_{\text {cat }}$.

Intersystem crossing occurs between the triplet state that correlates to $\mathrm{DPA}^{-}-\mathrm{O}_{2}$ and the singlet state that correlates diabatically with a DPA-O ${ }_{2}^{-}$state, i. e. it is associated to the charge transfer from DPA to $\mathrm{O}_{2}$ to form a superoxo anion. As this change of spin is associ- ated with a change in the electronic configuration, it does comply with the El-Sayed rules, and the strength of the spin-orbit coupling is above $70 \mathrm{~cm}^{-1}$, a relatively large value for a system that does not include heavy atoms.

For other spin-forbidden processes, it has been postulated that the rate for the spin-forbidden reaction is enhanced by the presence of a broad crossing region that maximizes the cross-over probability. That is not the case of the reaction between DPA-CoA and $\mathrm{O}_{2}$, for which this probability is maximized by the presence of a stable intermolecular well arising from the charge-dipole interaction that stabilizes the superoxo singlet state, lowering the energy of the minimum energy crossing point. For the formation of the peroxide a second charge transfer from DPA to $\mathrm{O}_{2}$ is required.

Our QM/MM calculations reveal that the minimum energy crossing point is the main reaction bottleneck. They also suggest that protonation of $\mathrm{O}_{2}$ takes place after formation of the peroxide, which is already stable in its deprotonated form. It could explain the absence of basic residues in the active site that could protonate $\mathrm{O}_{2}$ during its addition to the enolate. It is also relevant that the first molecule of the water-wire that connects the active site to the bulk changes its orientation following the formation of the peroxide. In their final configuration, they could easily capture the proton that could initiate the following step in the formation of DPGX. Finally, the comparison between the energy profiles calculated using different QM regions suggests that although the protein environment plays an important role in the diffusion of $\mathrm{O}_{2}$ towards the active site, and in the formation and stabilization of the enolate, it plays a passive role in the promotion of the spin-forbidden reaction. We believe that these results are general, and that similar behavior should be observed for other cofactorless spin-forbidden reactions that proceed via the formation of a peroxide.

\section{METHODS}

\section{A. Initial coordinates and molecular dynamics simulations}

The initial structure adopted in this work is based on the crystal structure of the DpgC/DPA-NH-CoA/O $\mathrm{O}_{2}$ complex (PDB: 5KAG, $2.68 \AA$ resolution). ${ }^{38}$ That crystal structure was obtained replacing the original substrate (DPA-CoA, see Fig.1) by the stable substrate ana$\log (\mathrm{DPA}-\mathrm{NH}-\mathrm{CoA})$ to prevent the reaction even in aerobic conditions. In our simulations, we exchanged DPANH-CoA by DPA-CoA while keeping $\mathrm{O}_{2}$ in its crystallographic position, a hydrophobic pocket less than $4 \AA$ away from $\mathrm{C}_{\alpha}$ of DPA, and the initial geometry was prepared using CHARMM-GUI. ${ }^{70,71}$ Hydrogen coordinates were generated with standard protonation states for all titrable residues using $\mathrm{CHARMM}^{72}$, and the protein complex was placed in the center of a cubic TIP3 water box large enough to include the protein and at least $10 \AA$ of solvent on all sides. Additionally, wa- 
ter molecules were randomly replaced by $\mathrm{K}^{+}$and $\mathrm{Cl}^{-}$ ions to ensure neutralization of the system, and provide an additional concentration of $0.15 \mathrm{M} \mathrm{KCl}$. The system was subject to classical MD equilibration of 10 $\mathrm{ns}$ at constant temperature $(\mathrm{T}=303.15 \mathrm{~K})$ and pressure (1 atm) using NAMD (http://www.ks.uiuc.edu/ Research/namd/), ${ }^{73}$ and CHARMM-36m forcefield. ${ }^{74,75}$ The Particle Mesh Ewald method was used for the electrostatics of the periodic boundary conditions. ${ }^{76}$ A time step of 2 fs was used with the ShakeH algorithm. ${ }^{77}$ To avoid $\mathrm{O}_{2}$ diffusion outside the active site, the positions of $\mathrm{O}_{2}$ and the non-hydrogen atoms of $\mathrm{DpgC}$ were fixed throughout the equilibration. The root-mean-square deviation (RMSD) with respect to the initial conformation was calculated for all the non-hydrogen protein atoms that were not located in any turn or coil (see Fig. S4) showing that the structure was well converged after $4 \mathrm{~ns}$, with an average RMSD of $1.56 \AA$.

To select an initial frame for the subsequent QM/MM calculations, we calculated the occupancy of water molecules in the active site following the same procedure used in Ref. ${ }^{78}$. Considering the last 5 ns of the MD trajectory, we calculated the averaged position of the water molecules within $7 \AA$ of the $\mathrm{O}_{2}$ molecule, and selected a frame for which the position of the waters was closed to their average position. Compared to the initial structure, the two water molecules crystallized around the active site kept their positions (although one of them exchanged with the solvent during the simulation time), while the active site recruited a third water molecule and a waterwire connecting the active site and the bulk was formed. A representation of the initial structure for QM/MM calculations is shown in Fig. S5.

\section{B. $\mathrm{QM} / \mathrm{MM}$ Calculations}

Once the system was classically equilibrated and the initial frame was selected, it was trimmed to a sphere of $26 \AA$ centered at the $\mathrm{S}$ atom of DPA-CoA. Atoms further away than $18 \AA$ of the $\mathrm{S}$ atom of DPA-CoA were kept frozen during all the QM/MM calculations. A full electrostatic embedding ${ }^{79}$ was adopted in all the calculations, using hydrogen link atoms to treat the QM/MM boundaries, maintaining the effect of the broken chemical bond between the QM and the MM region. QM/MM calculations were run coupling Q-Chem v5.2 ${ }^{80}$ and $\mathrm{CHARMM}^{72}$. QM calculations were carried out at the B3LYP/6-31G+(d,p) DFT level of theory for the ground singlet and triplet states. The D3 version of Grimme's dispersion correction was used. ${ }^{81,82}$

Initial optimization of the reactants $\left(\mathrm{DPA}^{-}+\mathrm{O}_{2}\right)$ and products $\left(\mathrm{DPA}-\mathrm{OO}^{-}\right.$) confirmed that the reactants were only stable in its triplet state, while the peroxide was only stable as a singlet. It also revealed that the protein prevents the bending of the DPA-OO- ${ }^{-}$peroxide, that remains almost planar. The reaction path was scanned by performing restrained geometry optimizations along the reaction coordinate that was defined as the energy difference between the ground triplet and singlet state, $\Delta E^{\text {Sing } / \text { Trip }}=E^{\text {triplet }}-E^{\text {singlet }}$, so the MECP is found for $\Delta E^{\text {Sing } / \text { Trip }}=0$. To impose that restrain, we added the following harmonic term to the potential:

$$
V=V_{0}+\frac{1}{2} K\left(\Delta E^{\mathrm{Sing} / \text { Trip }}-\Delta E_{i}^{\mathrm{Sing} / \text { Trip }}\right)^{2}
$$

where $V_{0}$ is the minimum value between $E^{\text {triplet }}$ and $E^{\text {singlet }}, K$ is the spring constant (whose value was set to $\left.500 \mathrm{eV}^{-1}\right), \Delta E_{i}^{\text {Sing/Trip }}$ is the reference value of $\Delta E^{\text {Sing/Trip }}$ to which the potential is biased, and $V$ is the resulting biased potential energy.

\section{Multireference calculations}

\section{1. $\mathrm{MRCl}$ calculations}

The reaction between S-Methyl-but-3-enethioate and $\mathrm{O}_{2}$ was simulated using the Multiconfiguration reference internally contracted configuration interaction method (MRCI), which captures the static correlation by the selection of all the electronic configurations that can be generated by the distribution of the electrons considered in the orbitals that belong to the active space. The dynamic correlation is then captured by optimizing variationally the best solution of a linear combination of all the electronic configurations generated by simple and double electronic excitations from all the reference configurations generated by the active space towards the orbitals of the virtual space. To account for the quadruple excitations and reduce the size-consistency error inherent to the method, Davidson correction has been applied.

Calculations were carried out using the MOLPRO package $^{83}$, and a $6-31 \mathrm{G}+(\mathrm{d}, \mathrm{p})$ basis set. For efficiency reasons, the geometries were first optimized at a DFT level using the B3LYP functional, and the D3 version of Grimme's dispersion correction, following by singlepoint MRCI calculations. This procedure is similar to the approach employed to shed light on the reactivity of metallic clusters ${ }^{84,85}$. The only difference is that here, the MECP was also optimized.

The procedure applied here is the following: First, the minimum crossing point (MECP) between triplet an singlet was optimized at the DFT level. Then, starting from that structure, the geometry was relaxed for different values of the reaction coordinate, which we assimilated to $\mathrm{C}_{\alpha}$-O distance $\left(r_{C_{\alpha} O}\right) \cdot r_{C_{\alpha} O}$ was sampled from $1.2 \AA$ to $7 \AA$, and taking as a reference value the $r_{C_{\alpha} O}$ distance at the MECP, for larger (smaller) $r_{C_{\alpha} O}$ values the geometry was optimized in its triplet (singlet) state. Furthermore, it allows a double-check to confirm that the electronic wavefunctions converge to the correct state, which is particularly tedious in the case of $\mathrm{O}_{2}$.

The energy of the geometries obtained at DFT level was recalculated using the internally contracted version 
of MRCI available in MOLPRO ${ }^{86,87}$. Within this method the SOC can be calculated rigorously using the BreitPauli spin-orbit operator. The first step required before runing MRCI calculations is the definition of the inicial wavefunction. In this work, the guess wavefaction was generated through a state-average CASSCF calculation $^{88,89}$ including in the active space four electrons in three orbitals: the two $\pi_{g}^{*}$ orbitals of $\mathrm{O}_{2}$ and the HOMO of S-Methyl-but-3-enethioate. In the stateaverage CASSCF wave function, one triplet and five singlet states were treated on an equal footing. This approach leads to stable optimized orbitals and configurations along the reaction path, which is required to obtain accurate and meaningful potential energy curves at a multireference level, which is hard to achieve for relatively large polyatomic systems. For the subsequent MRCI calculation, we computed the 9 states (three triplet and six singlet states) and the SOC between them. Under these conditions, the electronic wavefunctions of the triplet (singlets) states are constructed over a linear combination of about $6 \times 10^{6}\left(30 \times 10^{6}\right)$ contracted configurations.

Due to the relatively large number of coupled electronic states, avoided crossings in the adiabatic potential energy curves may impair their interpretation. In these cases, it may be useful to calculate the states in the diabatic representation, which also sheds light onto the nature of the electronic states involved. Since we are considering singlet and triplet states simultaneously, automatic diabatization algorithm cannot be employed, so we applied the diabatization model based on the geometric based approach $^{90}$, which has been successfully applied to diabatize highly entangled excited states of alkyl radicals ${ }^{91-93}$. It consists in a geometric approach where successive $2 \times 2$ diabatizations are performed between all coupled states iteratively. The validity of the approach was confirmed by the comparison between our diabatic curves are those obtained using the default molpro algorithm for the three triplet states.

\section{Semistochastic Heat-Bath Configuration Interaction}

The reaction between S-Methyl-but-3-enethioate and $\mathrm{O}_{2}$ was also simulated using the recent Semistochastic Heat-Bath Configuration Interaction (SHCI) method $^{66-68}$, which permits to overcome the critical limitation to the maximum number of orbitals that can be included in a multi-configurational self-consistent field (MCSCF) calculation (depending on the system up to 14-18 orbitals).

The SHCI algorithm consists of two stages. First, a variational wavefunction is computed using a set of iteratively selected determinants. These determinants are stochastically sampled, and they are filtered under a chosen energy value threshold $\left(\epsilon_{1}\right)$. The algorithm increases the number of determinants included in the set until the wavefunction has a constant and stable energy. In the second stage, the second-order correction to the variational energy is computed using multireference EpsteinNesbet perturbation theory.

The resulting energy is considered to be FullConfiguration Interaction (FCI) for a given active apace and basis set. To obtain the FCI energy, several calculations are computed for different values of $\epsilon_{1}$ and they are extrapolated to $\epsilon_{1} \rightarrow 0$. All the SHCI calculations were done in the open-source Python software package $\mathrm{PySCF}^{94}$, interfaced to the fast version of the algorithm, Arrow $^{95}$.

\section{ACKNOWLEDGMENTS}

The authors thank Prof. Enrique Verdasco for his support and help with the calculations. Funding by the Spanish Ministry of Science and Innovation (grants and PGC2018-096444-B-I00, and FIS2017-83473-C2-2-P) is also acknowledged. The authors acknowledge funding by Fundación Salamanca City of Culture and Knowledge (Programme for attracting scientific talent to Salamanca).

${ }^{1}$ H. D. Holland, Phil. Trans. R. Soc. B 361, 903 (2006).

${ }^{2} \mathrm{R}$. Ligrone, "The great oxygenation event," in Biological Innovations that Built the World: A Four-billion-year Journey through Life and Earth History (Springer International Publishing, Cham, 2019) pp. 129-154.

${ }^{3}$ C. W. Machan, ACS Catal. 10, 2640 (2020).

${ }^{4}$ M. L. Pegis, C. F. Wise, D. J. Martin, and J. M. Mayer, Chem. Rev. 118, 2340 (2018).

${ }^{5}$ C. Feng, M. B. Faheem, J. Fu, Y. Xiao, C. Li, and Y. Li, ACS Catal. 10, 4019 (2020).

${ }^{6}$ T. D. Bugg, Tetrahedron 59, 7075 (2003).

${ }^{7}$ S. Sahu and D. P. Goldberg, J. Am. Chem. Soc. 138, 11410 (2016).

${ }^{8}$ A. J. Jasniewski and L. Que, Chem. Rev. 118, 2554 (2018).

${ }^{9}$ W. Zhang, W. Lai, and R. Cao, Chem. Rev. 117, 3717 (2017).

${ }^{10}$ C. E. Elwell, N. L. Gagnon, B. D. Neisen, D. Dhar, A. D. Spaeth, G. M. Yee, and W. B. Tolman, Chem. Rev. 117, 2059 (2017).

${ }^{11}$ M. Wikstrom, K. Krab, and V. Sharma, Chem. Rev. 118, 2469 (2018).

${ }^{12}$ E. C. Kisgeropoulos, J. J. Griese, Z. R. Smith, R. M. M. Branca, C. R. Schneider, M. Högbom, and H. S. Shafaat, J. Am. Chem. Soc. 142, 5338 (2020).

${ }^{13}$ S. S. Chaturvedi, R. Ramanan, N. Lehnert, C. J. Schofield, T. G. Karabencheva-Christova, and C. Z. Christov, ACS Catal. 10, 1195 (2020).

${ }^{14}$ R. Suardiaz, P. G. Jambrina, L. Masgrau, A. González-Lafont, E. Rosta, and J. M. Lluch, J. Chem. Theory Comput. 4, 2079 (2016).

${ }^{15}$ K. P. Jensen and U. Ryde, J. Biol. Chem. 279, 14561 (2004).

${ }^{16}$ A. Cebrián-Prats, n. González-Lafont, and J. M. Lluch, ACS Catalysis 10, 138 (2020).

${ }^{17}$ C. M. Marian, WIREs Comput Mol Sci 2, 187 (2012).

${ }^{18}$ G. Baryshnikov, B. Minaev, and H. Ågren, Chem. Rev. 117, 6500 (2017).

${ }^{19}$ P. Chaiyen, M. W. Fraaije, and A. Mattevi, Trends Biochem Sci. 37, 373 (2012).

${ }^{20}$ A. Mattevi, Trends Biochem Sci. 31, 276 (2006).

${ }^{21}$ E. Romero, J. R. Gómez Castellanos, G. Gadda, M. W. Fraaije, and A. Mattevi, Chem. Rev. 118, 1742 (2018).

${ }^{22}$ S. Thierbach, N. Bui, J. Zapp, S. Chhabra, R. Kappl, and S. Fetzner, Chem. Biol. 21, 217 (2014).

${ }^{23}$ S. Bui and R. A. Steiner, Curr Opin Struct Biol. 41, 109 (2016). 
${ }^{24}$ X. Fretzner and R. A. Steiner, Appl Microbiol Biotechnol. 4186 791 (2010).

${ }^{25}$ L. Gabison, C. Chopard, N. Colloc'h, F. Peyrot, B. Castro, M. E. Hajji, M. Altarsha, G. Monard, M. Chiadmi, and T. Prangé, Proteins 79, 1964 (2011).

${ }^{26}$ D. Wei, X. Huang, Y. Qiao, J. Rao, L. Wang, F. Liao, and C.-G. Zhan, ACS Catal. 7, 4623 (2017).

${ }^{27}$ S. Bui, D. von Stetten, P. G. Jambrina, T. Prange, N. Colloc'h, D. de Sanctis, A. Royant, E. Rosta, and R. A. Steiner, Angew. Chem. Int. Ed. 53, 13710 (2014).

${ }^{28}$ S. Visitsatthawong, P. Chenprakhon, P. Chaiyen, and P. Surawatanawong, J. Am. Chem. Soc. 137, 9363 (2015).

${ }^{29}$ P. J. Silva, PeerJ 4, e2805 (2016).

${ }^{30}$ F. G. Cantu Reinhard, J. L. DuBois, and S. P. de Visser, J. Phys. Chem. B 122, 10841 (2018)

${ }^{31}$ B. Minaev, Chem. Phys. 521, 61 (2019).

${ }^{32}$ R. Prabhakar, P. E. Siegbahn, and B. F. Minaev, Biochim. Biophys. Acta 1647, 173 (2003).

${ }^{33}$ R. Prabhakar, P. E. M. Siegbahn, B. F. Minaev, and H. Ågren, J. Phys. Chem. B 106, 3742 (2002).

${ }^{34}$ M. M. Machovina, R. J. Usselman, and J. L. DuBois, J. Biol. Chem. 291, 17816 (2016).

${ }^{35}$ A. Hernández-Ortega, M. G. Quesne, S. Bui, D. J. Heyes, R. A. Steiner, N. S. Scrutton, and S. P. de Visser, J. Am. Chem. Soc. 137, 7474 (2015).

${ }^{36}$ S. Fetzner, Nature 447, 342 (2007).

${ }^{37}$ S. Fetzner, Nat. Chem. Biol. 3, 374 (2007).

${ }^{38} \mathrm{~K}$. Li, E. N. Fielding, H. L. Condurso, and S. D. Bruner, Acta Cryst. D73, 573 (2017).

${ }^{39}$ C. T. Lohans, D. Y. Wang, J. Wang, R. B. Hamed, and C. J. Schofield, ACS Catal. 7, 6587 (2017).

${ }^{40}$ E. N. Fielding, P. F. Widboom, and S. D. Bruner, Biochem. 46, 13994 (2007).

${ }^{41}$ P. F. Widboom and S. D. Bruner, ChemBioChem 10, 1757 (2009).

${ }^{42}$ C. C. Tseng, F. H. Vaillancourt, S. D. Bruner, and C. T. Walsh, Chemistry \& Biology 11, 1195 (2004).

${ }^{43}$ N. V. Di Russo, H. L. Condurso, K. Li, S. D. Bruner, and A. E. Roitberg, Chem. Sci. 6, 6341 (2015).

${ }^{44}$ D. S. Kaliakin, D. G. Fedorov, Y. Alexeev, and S. A. Varganov, J. Chem. Theory and Comput. 15, 6074 (2019).

${ }^{45}$ A. O. Lykhin, D. S. Kaliakin, G. E. dePolo, A. A. Kuzubov, and S. A. Varganov, Int. J. Quantum Chem. 116, 750 (2016).

${ }^{46}$ A. O. Lykhin and S. A. Varganov, Phys. Chem. Chem. Phys. 22, $5500(2020)$

${ }^{47}$ J. N. Harvey, WIREs Comput Mol Sci 4, 1 (2014)

${ }^{48}$ J. N. Harvey, Faraday Discuss. 127, 165 (2004).

49 J. N. Harvey, Phys. Chem. Chem. Phys. 9, 331 (2007)

${ }^{50}$ C. Zener and R. H. Fowler, Proc. R. Soc. Lond. 137, 696 (1932).

${ }^{51}$ G. E. dePolo, D. S. Kaliakin, and S. A. Varganov, J. Phys. Chem. A 120, 8691 (2016).

${ }^{52}$ P. J. Silva and M. J. Ramos, J. Phys. Chem. B 111, 12883 (2007).

${ }^{53}$ P. J. Silva and M. J. Ramos, Bioorg. Med. Chem. 16, 2726 (2008).

${ }^{54}$ S. Gomez-Carrasco, L. Gonzalez-Sanchez, A. Aguado, O. Roncero, J. M. Alvarino, M. L. Hernandez, and M. Paniagua, J. Chem. Phys. 121, 4605 (2004)

${ }^{55}$ C. Sanz-Sanz, O. Roncero, M. Paniagua, and A. Aguado, J. Chem. Phys. 139, 184302 (2004).

${ }^{56}$ A. Zanchet, F. Lique, O. Roncero, J. R. Goicoechea, and N. Bulut, A\&A 626, A103 (2019).

${ }^{57}$ A. Zanchet, M. Menendez, P. G. Jambrina, and F. J. Aoiz, J. Chem. Phys. 151, 094307 (2019).

${ }^{58}$ C. Sanz-Sanz and G. A. Worth, Phys. Chem. Chem. Phys. 21, 14429 (2019)

${ }^{59}$ C. Sanz-Sanz, A. Aguado, O. Roncero, and F. Naumkin, J Chem. Phys. 143, 234303 (2015).

${ }^{60}$ G. Herzberg, Spectra of Diatomic Molecules (Van Nostrand Reinhold, New York, 1950).

${ }^{61}$ M. A. El-Sayed, J. Chem. Phys. 38, 2834 (1963).
${ }^{62}$ H. Li, A. Kamasah, S. Matsika, and A. G. Suits, Nat. Chem. 11, 123 (2019).

${ }^{63}$ C. Schweitzer and R. Schmidt, Chem. Rev. 103, 1685 (2003).

${ }^{64}$ G. F. Minaev, Int. J. Quantum Chem. 12, 367 (1980).

${ }^{65}$ K. M. Ervin, I. Anusiewicz, P. Skurski, J. Simons, and W. C. Lineberger, J. Phys. Chem. A 107, 8521 (2003).

${ }^{66} \mathrm{~S}$. Sharma, A. A. Holmes, G. Jeanmairet, A. Alavi, and C. J. Umrigar, J. Chem. Theory Comput. 13, 1595 (2017).

${ }^{67}$ A. A. Holmes, N. M. Tubman, and C. J. Umrigar, J. Chem. Theory Comput. 12, 3674 (2016).

68 J. E. T. Smith, B. Mussard, A. A. Holmes, and S. Sharma, J. Chem. Theory Comput. 13, 5468 (2017).

${ }^{69}$ S. Visitsatthawong, P. Chenprakhon, P. Chaiyen, and P. Surawatanawong, J. Am. Chem. Soc. 137, 9363 (2015).

${ }^{70}$ S. Jo, T. Kim, V. G. Iyer, and W. Im, J. Comput. Chem. 29, 1859 (2008).

${ }^{71}$ J. Lee, X. Cheng, J. M. Swails, M. S. Yeom, P. K. Eastman, J. A. Lemkul, S. Wei, J. Buckner, J. C. Jeong, Y. Qi, S. Jo, V. S. Pande, D. A. Case, C. L. Brooks, A. D. MacKerell, J. B. Klauda, and W. Im, J. Chem. Theory Comput. 12, 405 (2016).

${ }^{72}$ B. R. Brooks, C. L. Brooks III, A. D. Mackerell Jr., L. Nilsson, R. J. Petrella, B. Roux, Y. Won, G. Archontis, C. Bartels, S. Boresch, A. Caflisch, L. Caves, Q. Cui, A. R. Dinner, M. Feig, S. Fischer, J. Gao, M. Hodoscek, W. Im, K. Kuczera, T. Lazaridis, J. Ma, V. Ovchinnikov, E. Paci, R. W. Pastor, C. B. Post, J. Z. Pu, M. Schaefer, B. Tidor, R. M. Venable, H. L. Woodcock, X. Wu, W. Yang, D. M. York, and M. Karplus, J. Comput. Chem. 30, 1545 (2009).

${ }^{73}$ J. C. Phillips, R. Braun, W. Wang, J. Gumbart, E. Tajkhorshid, E. Villa, C. Chipot, R. D. Skeel, L. Kalé, and K. Schulten, J. Comput. Chem. 26, 1781 (2005).

${ }^{74}$ J. Huang, S. Rauscher, G. Nawrocki, T. Ran, M. Feig, B. L. de Groot, H. Grubmuller, and A. D. MacKerell, Nat. Methods 14, 71 (2017)

${ }^{75}$ A. D. MacKerell, M. Feig, and C. L. Brooks, J. Am. Chem. Soc. 126, 698 (2004).

${ }^{76}$ T. Darden, D. York, and L. Pedersen, J. Chem. Phys. 98, 10089 (1993).

77 J.-P. Ryckaert, G. Ciccotti, and H. J. Berendsen, J. Comput. Phys. 23, 327 (1977).

${ }^{78}$ V. Sharma, P. G. Jambrina, M. Kaukonen, E. Rosta, and P. R. Rich, Proc. Nat. Ac. Sci. 114, E10339 (2017).

${ }^{79}$ H. L. Woodcock III, M. Hodoscek, A. T. B. Gilbert, P. M. W. Gill, H. F. Schaefer III, and B. R. Brooks, J. Comput. Chem. 28, 1485 (2007).

${ }^{80}$ Y. Shao, Z. Gan, E. Epifanovsky, A. T. Gilbert, M. Wormit, J. Kussmann, A. W. Lange, A. Behn, J. Deng, X. Feng, D. Ghosh, M. Goldey, P. R. Horn, L. D. Jacobson, I. Kaliman, R. Z. Khaliullin, T. Kuś, A. Landau, J. Liu, E. I. Proynov, Y. M. Rhee, R. M. Richard, M. A. Rohrdanz, R. P. Steele, E. J. Sundstrom, H. L. W. III, P. M. Zimmerman, D. Zuev, B. Albrecht, E. Alguire, B. Austin, G. J. O. Beran, Y. A. Bernard, E. Berquist, K. Brandhorst, K. B. Bravaya, S. T. Brown, D. Casanova, C.M. Chang, Y. Chen, S. H. Chien, K. D. Closser, D. L. Crittenden, M. Diedenhofen, R. A. D. Jr., H. Do, A. D. Dutoi, R. G. Edgar, S. Fatehi, L. Fusti-Molnar, A. Ghysels, A. GolubevaZadorozhnaya, J. Gomes, M. W. Hanson-Heine, P. H. Harbach, A. W. Hauser, E. G. Hohenstein, Z. C. Holden, T.-C. Jagau, H. Ji, B. Kaduk, K. Khistyaev, J. Kim, J. Kim, R. A. King, P. Klunzinger, D. Kosenkov, T. Kowalczyk, C. M. Krauter, K. U. Lao, A. D. Laurent, K. V. Lawler, S. V. Levchenko, C. Y. Lin, F. Liu, E. Livshits, R. C. Lochan, A. Luenser, P. Manohar, S. F. Manzer, S.-P. Mao, N. Mardirossian, A. V. Marenich, S. A. Maurer, N. J. Mayhall, E. Neuscamman, C. M. Oana, R. OlivaresAmaya, D. P. O'Neill, J. A. Parkhill, T. M. Perrine, R. Peverati, A. Prociuk, D. R. Rehn, E. Rosta, N. J. Russ, S. M. Sharada, S. Sharma, D. W. Small, A. Sodt, T. Stein, D. Stück, Y.-C. Su, A. J. Thom, T. Tsuchimochi, V. Vanovschi, L. Vogt, O. Vydrov, T. Wang, M. A. Watson, J. Wenzel, A. White, C. F. Williams, J. Yang, S. Yeganeh, S. R. Yost, Z.-Q. You, I. Y. 
Zhang, X. Zhang, Y. Zhao, B. R. Brooks, G. K. Chan, D. M. Chipman, C. J. Cramer, W. A. G. III, M. S. Gordon, W. J. Hehre, A. Klamt, H. F. S. III, M. W. Schmidt, C. D. Sherrill, D. G. Truhlar, A. Warshel, X. Xu, A. Aspuru-Guzik, R. Baer, A. T. Bell, N. A. Besley, J.-D. Chai, A. Dreuw, B. D. Dunietz, T. R. Furlani, S. R. Gwaltney, C.-P. Hsu, Y. Jung, J. Kong, D. S. Lambrecht, W. Liang, C. Ochsenfeld, V. A. Rassolov, L. V. Slipchenko, J. E. Subotnik, T. V. Voorhis, J. M. Herbert, A. I. Krylov, P. M. Gill, and M. Head-Gordon, Mol. Phys. 113, 184 (2015).

${ }^{81}$ S. Grimme, J. Antony, S. Ehrlich, and H. Krieg, J. Chem. Phys. 132, 154104 (2010).

${ }^{82}$ S. Grimme, S. Ehrlich, and L. Goerigk, J. Comput. Chem. 32, 1456 (2011).

${ }^{83}$ H.-J. Werner, P. J. Knowles, G. Knizia, F. R. Manby, M. Schütz, P. Celani, W. Györffy, D. Kats, T. Korona, R. Lindh, A. Mitrushenkov, G. Rauhut, K. R. Shamasundar, T. B. Adler, R. D. Amos, A. Bernhardsson, A. Berning, D. L. Cooper, M. J. O. Deegan, A. J. Dobbyn, F. Eckert, E. Goll, C. Hampel, A. Hesselmann, G. Hetzer, T. Hrenar, G. Jansen, C. Köppl, Y. Liu, A. W. Lloyd, R. A. Mata, A. J. May, S. J. McNicholas, W. Meyer, M. E. Mura, A. Nicklass, D. P. O'Neill, P. Palmieri, D. Peng, K. Pflüger, R. Pitzer, M. Reiher, T. Shiozaki, H. Stoll, A. J. Stone, R. Tarroni, T. Thorsteinsson, and M. Wang, "Molpro, version 2015.1, a package of ab initio programs," (2015), see http://www.molpro.net.
${ }^{84}$ A. Zanchet, A. Dorta-Urra, A. Aguado, and O. Roncero, J. Phys. Chem. C 115, 47 (2011).

${ }^{85}$ A. Zanchet, P. Lopez-Caballero, A. O. Mitrushchenkov, D. Buceta, M. A. Lopez-Quintela, A. W. Hauser, and M. P. de LaraCastells, J. Phys. Chem. C 123, 27064 (2019).

${ }^{86}$ H.-J. Werner and P. J. Knowles, J. Chem. Phys. 89, 5803 (1988).

${ }^{87}$ P. J. Knowles and H.-J. Werner, Chem. Phys. Lett. 145, 514 (1988).

${ }^{88}$ H.-J. Werner and P. J. Knowles, J. Chem. Phys. 82, 5053 (1985).

${ }^{89}$ P. J. Knowles and H.-J. Werner, Chem. Phys. Lett. 115, 259 (1985).

${ }^{90}$ A. Zanchet, L. Bañares, M. L. Senent, and A. Garcia-Vela, Phys. Chem. Chem. Phys. 18, 33195 (2016).

${ }^{91}$ G. Balerdi, J. Woodhouse, A. Zanchet, R. de Nalda, M. L. Senent, A. Garcia-Vela, and L. Bañares, Phys. Chem. Chem. Phys. 18, 110 (2016).

${ }^{92}$ S. M. Poullain, D. V. Chicharro, A. Zanchet, M. G. Gonzalez, L. Rubio-Lago, and L. Bañares, Phys. Chem. Chem. Phys. 18, 17054 (2016).

${ }^{93}$ D. V. Chicharro, S. M. Poullain, A. Zanchet, A. Bouallagui, A. Garcia-Vela, M. L. Senent, L. Rubio-Lago, and L. Bañares, Chem. Sci. 10, 6494 (2019).

${ }^{94}$ Q. Sun, T. C. Berkelbach, N. S. Blunt, G. H. Booth, S. Guo, Z. Li, J. Liu, J. D. McClain, E. R. Sayfutyarova, S. Sharma, S. Wouters, and G. K. Chan, Wiley Interdiscip. Rev. Comput. Mol. Sci. 8, e1340 (2017).

${ }^{95} \mathrm{~J}$. Li, M. Otten, A. A. Holmes, S. Sharma, and C. J. Umrigar, J. Chem. Phys. 148, 214110 (2018). 